\title{
Do número de Milnor ao número de Milnor de Lê
}

\author{
Camila Mariana Ruiz
}




\title{
Do número de Milnor ao número de Milnor de Lê
}

\author{
Camila Mariana Ruiz
}

\section{Orientador: Prof. Dr. Nivaldo de Góes Grulha Júnior}

Dissertação apresentada ao Instituto de Ciências Matemáticas e de Computação - ICMC-USP, como parte dos requisitos para obtenção do título de Mestre em Ciências - Matemática . VERSÃO REVISADA

USP - São Carlos

Julho de 2011 
Ficha catalográfica elaborada pela Biblioteca Prof. Achille Bassi e Seção Técnica de Informática, ICMC/USP, com os dados fornecidos pelo(a) autor(a)

Ruiz, Camila Mariana
Do número de Milnor ao número de Milnor de Lê /
Camila Mariana Ruiz; orientador Nivaldo de Góes
Grulha Júnior -- São Carlos, 2011.
90 p.
Dissertação (Mestrado - Programa de Pós-Graduação en
Matemática) -- Instituto de Ciências Matemáticas e
de Computação, Universidade de São Paulo, 2011.
1. Buquê de esferas. 2. Estratificação. 3. Número
de Milnor. 4. Número de Milnor de Lê. 5. Variedades
Singulares . I. de Góes Grulha Júnior, Nivaldo,
orient. II. Título.


À minha mãe Eliana e à minha avó Rosa.

Às minhas tias Elizabete e Elza. 
"O correr da vida embrulha tudo, a vida é assim: esquenta e esfria, aperta e daí afrouxa, sossega e depois desinquieta. O que ela quer da gente é coragem. O que Deus quer é ver a gente aprendendo a ser capaz de ficar alegre a mais, no meio da alegria, e inda mais alegre ainda no meio da tristeza! Só assim de repente, na horinha em que se quer, de propósito - por coragem. Será? Era o que eu às vezes achava. Ao clarear do dia."

João Guimarães Rosa, Grande Sertão: Veredas. 


\section{Agradecimentos}

Agradeço à Deus, por caminhar comigo.

À minha família, sem a qual eu nada seria.

Ao Felipe, pelo carinho e bom humor de sempre e pelo apoio nos momentos mais difíceis. Obrigada Fe.

Ao Nivaldo, meu querido orientador e amigo, pela enorme paciência, humildade, dedicação e pelo apoio de sempre.

À querida professora Cidinha, por ter sido uma professora de Cálculo I tão motivante e por ter me orientado durante a graduação.

Ao professor Carlos Biasi, pela amizade e por suas aulas inesquecíveis.

Ao professor Jean-Paul Brasselet, que mesmo em uma visita tão rápida ao Brasil e com tantos compromissos, encontrou tempo para responder as minhas perguntas com atenção e paciência.

Ao professor Lê Dung Tráng, que foi muito atencioso ao responder minhas dúvidas tão prontamente.

À Thaís pela amizade e pela ajuda de todos os dias.

A todos os meus amigos que se tornaram minha família aqui em São Carlos;

Aos professores e funcionários do ICMC que direta ou indiretamente contribuíram para mais esta conquista.

Aos meus professores do colégio, Adevaldo e Arnaldo, pelo comprometimento com seus alunos e paixão pela educação. 
À Fapesp e à Capes pelo apoio financeiro para realização deste trabalho. 


\section{Resumo}

Neste trabalho, apresentamos um breve compêndio sobre o estudo topológico das fibras de Milnor. Abordamos o caso clássico, estudado por J. Milnor, e a generalização apresentada por Lê D. T. para o caso de germes de funções analíticas definidas em variedades singulares. Nestas duas situações, os resultados principais tratam de germes de funções com singularidades isoladas. 



\section{Abstract}

In this work, we present a brief compendium about the topological study of J. Milnor fibers. We address the classic case, studied by Milnor, and the generalization presented by Lê D. T. for the case of germs of analytic functions defined on singular varieties. In both situations, the main results deal with germs of functions with isolated singularities. 



\section{Sumário}

$\begin{array}{ll}\text { Introdução } & 11\end{array}$

$\begin{array}{lll}1 & \text { Preliminares } & 15\end{array}$

$1.1 \quad$ Variedades diferenciáveis . . . . . . . . . . . . . . . . . . . . . . . . . 15

1.2 Homotopia . . . . . . . . . . . . . . . . . . . . . . . . . . . . . . . . . 19

1.3 Homotopia de pares e homotopia relativa . . . . . . . . . . . . . . . . . 22

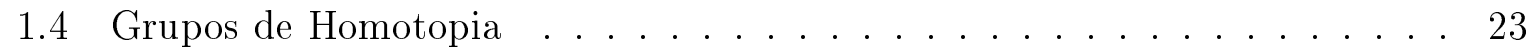

$1.4 .1 \quad$ O Grupo Fundamental . . . . . . . . . . . . . . . . . . . . . . . . . 23

1.5 Homologia singular . . . . . . . . . . . . . . . . . . . . 24

$1.6 \quad$ Complexos $C W$. . . . . . . . . . . . . . . . . . . . . . . . . . . . . . . . . . . . . 29

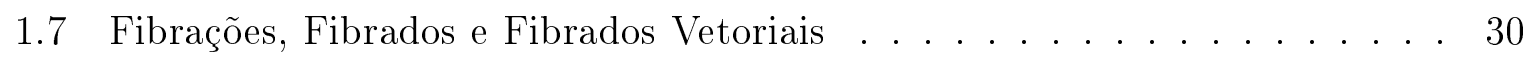

1.8 Teoria de singularidades $\ldots \ldots \ldots$. . . . . . . . . . . . . . . . . . . . . . 32

1.9 Variedades algébricas e espaços analíticos complexos . . . . . . . . . . . . . 33

1.10 Estratificação de Whitney . . . . . . . . . . . . . . . . . . . 36

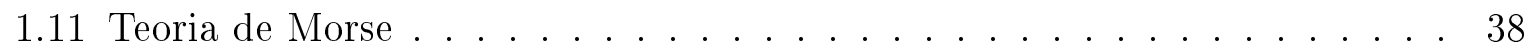

\begin{tabular}{|lll}
2 & O Teorema da Fibração & 41
\end{tabular}

\begin{tabular}{lll|}
\hline 3 & A Topologia das Fibras & 61
\end{tabular}

4 A Topologia das Fibras para o caso de um ponto crítico isolado 71 
5 Funções Analíticas Complexas com Singularidades Isoladas

5.1 Funções com singularidades isoladas . . . . . . . . . . . . . . . . . . . . . . 79

5.2 Morsificação . . . . . . . . . . . . . . . . . . . . . 82

5.3 Link Complexo e Profundidade . . . . . . . . . . . . . . . . . . . . . 84

$5.3 .1 \quad$ O Link Complexo . . . . . . . . . . . . . . . . . . . . . . . . 84

5.3 .2 Profundidade homotópica retificada . . . . . . . . . . . . . . . 85

5.4 O número de Milnor de Lêt . . . . . . . . . . . . . . . . . . . . . . . . . 87 


\section{Introdução}

Em [19] J. Milnor estudou a topologia dos pontos singulares de uma hipersuperfície complexa. Ele obteve um resultado impressionante sobre pontos singulares isolados, a saber, seja $f:\left(\mathbb{C}^{n}, 0\right) \longrightarrow(\mathbb{C}, 0)$ um germe de função analítica complexa, se 0 for um ponto crítico isolado de um representante (suficientemente pequeno) do germe, então definida a função $\phi: S_{\varepsilon} \backslash K \longrightarrow S^{1}$, dada por $\phi=f /|f|$, em que $f$ é polinomial complexa não nula com $f(0)=0$ e $K=S_{\varepsilon} \cap f^{-1}(0),\left(\phi, S_{\varepsilon} \backslash K, S^{1}\right)$ é um fibrado diferenciável tal que as fibras de $f$ em uma vizinhança da origem, chamadas fibras de Milnor de $f$ em 0, tem o tipo de homotopia de um buquê de esferas de dimensão real igual à dimensão complexa da fibra. O número de esferas do buquê, normalmente denotado por $\mu(f)$, é chamado de número de Milnor. O número de Milnor é um importante invariante de um germe de função analítica com ponto crítico isolado.

Este invariante fornece várias informações sobre a geometria de $f$, e está relacionado a outros ramos da matemática, por exemplo, no caso em que $f$ tem ponto crítico isolado na origem, os seguintes invariantes, que coincidem a menos de sinal:

a) O número de Milnor de $f$ em 0 , denotado por $\mu(f)$;

b) O número de pontos de Morse de uma Morsificação de $f$;

c) O índice de Poincaré-Hopf do campo gradiente de $f$ conjugado $\overline{\nabla f}$.

Desta maneira, os estudos desenvolvidos por J. Milnor em [19] passaram a desempenhar um papel fundamental para a teoria de singularidades, não só do ponto de vista topológico, mas também do ponto de vista algébrico e geométrico. 
H. Hamm em sua tese de doutorado [7] mostrou que um resultado semelhante aos obtidos por J. Milnor também é válido para interseções completas com singularidade isolada. Em [15], Lê D. T. mostrou que a fibra de Milnor da restrição de uma forma linear genérica sobre uma interseção completa, possivelmente com singularidades não isoladas, tem o tipo de homotopia de um buquê de esferas de dimensão média.

Em [12], Lê D. T. observou que o resultado de [15] mencionado acima implica um resultado similar ao de J. Milnor, que $f:(X, 0) \longrightarrow(\mathbb{C}, 0)$ um germe de uma função analítica complexa com uma singularidade isolada definido sobre $(X, 0)$, um germe de interseção completa, tem fibras de Milnor com o tipo de homotopia de um buquê de esferas de dimensão real igual à dimensão complexa da fibra.

Finalmente em [13], Lê D. T. buscou um conjunto maior de espaços em que um resultado semelhante ao de Milnor se verifica. Estes foram denominados espaços de Milnor, e comportam os espaços nos quais a profundidade homotópica retificada é máxima, isto é, igual à dimensão do espaço. Neste caso, Lê D. T. provou que a fibra genérica da função $f$ definida em um espaço de Milnor tem o tipo de homotopia de um buquê de esferas. E definimos então o número de esferas do buquê como sendo o número de Milnor de Lê, denotado por $\mu_{L}(f)$.

Um dos objetivos desta dissertação é apresentar parte dos estudos de Milnor descritos em [19], como a construção da fibração de Milnor e o estudo topológico das fibras desta fibração. Pretendemos expor também, a generalização dos resultados de Milnor apresentada por Lê D. T. em [13] para germes de funções holomorfas com singularidade isolada definidas em variedades singulares.

No Capítulo 1, introduzimos alguns conceitos básicos que serão utilizados ao longo do texto.

Nos Capítulos 2 a 4 foram desenvolvidos os estudos referentes ao Teorema da Fibração de Milnor: no Capítulo 2 apresentamos o Teorema da Fibração que prova que uma determinada aplicação $\phi$ é um fibrado diferenciável localmente trivial. Conhecendo o fibrado fornecido pela aplicação $\phi$, no Capítulo 3 nos dedicamos ao estudo da topologia das fibras $F_{\theta}$ desta fibração. Já no Capítulo 4 , consideramos a hipótese adicional de uma função $f$ com singularidade isolada na origem de $\mathbb{C}^{n+1}$ e, mais uma vez estudamos a topologia das 
fibras da fibração $\phi$ neste caso. Isto conclui nossa exposição sobre parte dos resultados de J. Milnor apresentados em [19].

Por fim, no Capítulo 5, apresentamos os principais resultados do trabalho [13] de Lê D. T. mencionado anteriormente, que expõe uma generalização dos resultados de J. Milnor para germes de funções holomorfas com singularidade isolada definidas sobre variedades singulares. 



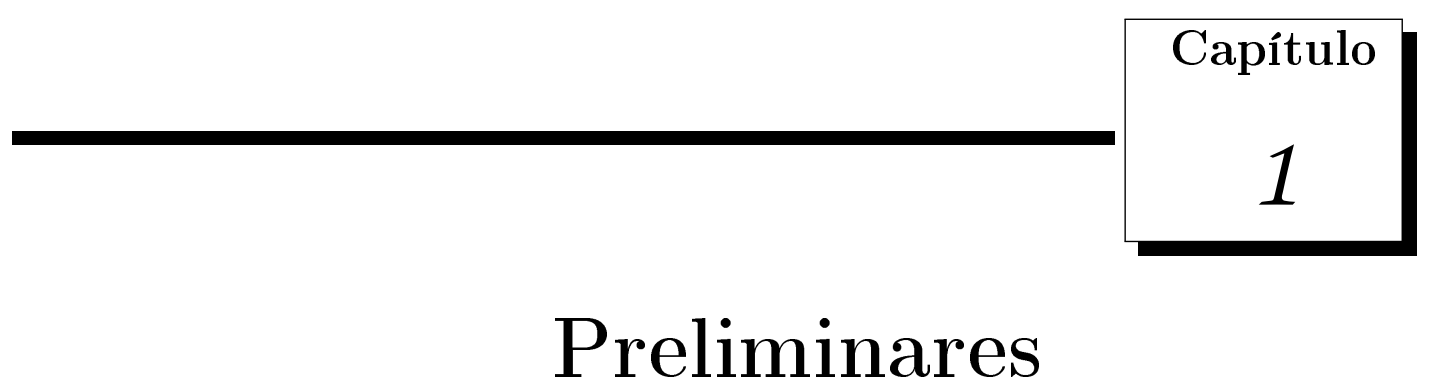

\subsection{Variedades diferenciáveis}

Definição 1.1.1. Seja $M$ um espaço topológico. Um sistema de coordenadas locais ou uma carta em $M$ é um homeomorfismo $\varphi: U \rightarrow \varphi(U)$ de um subconjunto aberto $U \subset M$ sobre um aberto $\varphi(U) \subset \mathbb{R}^{m}$.

Definição 1.1.2. Um atlas $\mathcal{U} m$-dimensional de um espaço topológico $M$ é uma família de sistemas de coordenadas locais $\varphi: U \rightarrow \varphi(U) \subset \mathbb{R}^{m}$, tal que os domínios dos sistemas de coordenadas cubram $M$.

Definição 1.1.3. Dados os sistemas de coordenadas locais $\varphi: U \rightarrow \mathbb{R}^{m}$ e $\psi: V \rightarrow \mathbb{R}^{m}$ no espaço topológico $M$, tais que $U \cap V \neq \emptyset$, então o homeomorfismo

$$
\phi_{\varphi \psi}=\psi \circ \varphi^{-1}: \varphi(U \cap V) \rightarrow \psi(U \cap V)
$$

é chamado mudança de coordenadas.

Observação 1.1.1. Seja a mudança de coordenadas $\phi_{\varphi \psi}=\psi \circ \varphi^{-1}$, então $\left(\psi \circ \varphi^{-1}\right)^{-1}=$ $\varphi \circ \psi^{-1}=\phi_{\psi \varphi}$ é também uma mudança de coordenadas.

Definição 1.1.4. Um atlas $\mathcal{U}$ em um espaço topológico $M$ é diferenciável de classe $C^{k}$, se todas as mudanças de coordenadas $\phi_{\varphi \psi}$ forem aplicações diferenciáveis de classe $C^{k}$. 
Observação 1.1.2. Da Observação 1.1.1 e da Definição 1.1.4 segue que em um atlas diferenciável $\mathcal{U}$ todas as mudanças de coordenadas $\phi_{\varphi \psi}$ são difeomorfismos.

Definição 1.1.5. Uma variedade diferenciável $m$-dimensional, de classe $C^{k}$, é um par $(M, \mathcal{U})$, em que $M$ é um espaço topológico Hausdorff com base enumerável e $\mathcal{U}$ é um atlas $m$-dimensional de classe $C^{k}$.

Como vimos na Observação 1.1 .2 em um atlas diferenciável $\mathcal{U}$ todas as mudanças de coordenadas $\phi_{\varphi \psi}$ são difeomorfismos. É exatamente esta propriedade que nos permite transportar todas as noções de Cálculo Diferencial do $\mathbb{R}^{m}$ para as variedade diferenciáveis.

Seja $M$ uma variedade de classe $C^{k}$ e seja $p$ um ponto de $M$. Indicamos por $C_{p}$ o conjunto de todos os caminhos $\lambda: I \rightarrow M$, definidos num intervalo aberto $I$, contendo 0 , tais que $\lambda(0)=p$ e $\lambda$ é diferenciável em 0 . Diremos que dois caminhos $\lambda, \mu \in C_{p}$ são equivalentes, e escreveremos $\lambda \sim \mu$, quando existir um sistema de coordenadas locais $\varphi: U \rightarrow \mathbb{R}^{m}$ em $M$, com $p \in U$, tal que $(\varphi \circ \lambda)^{\prime}(0)=(\varphi \circ \mu)^{\prime}(0)$. Como as mudanças de coordenadas são difeomorfismo, a igualdade $(\varphi \circ \lambda)^{\prime}(0)=(\varphi \circ \mu)^{\prime}(0)$ será verdadeira para todo sistema de coordenadas $\varphi: U \rightarrow \mathbb{R}^{m}$ em $M, p \in U$. Resulta daí que a relação acima é uma relação de equivalência.

O vetor velocidade $\lambda^{\prime}$ de um caminho $\lambda \in C_{p}$ é, por definição, a classe de equivalência de $\lambda$. Ou seja, $\lambda^{\prime}=\left\{\mu \in C_{p}: \mu \sim \lambda\right\}$.

Definição 1.1.6. Definimos o espaço tangente à variedade $M$ no ponto $p$ como sendo o conjunto quociente $C_{p} / \sim$. Denotaremos o espaço tangente à variedade $M$ no ponto $p$ por $T_{p} M$.

Podemos mostrar que o espaço tangente a uma variedade diferenciável $M$ em um ponto $p \in M$ é um espaço vetorial de mesma dimensão que $M$.

Dada uma variedade diferenciável $m$-dimensional $M$, podemos obter uma nova variedade $2 m$-dimensional a partir de uma "união" dos espaços tangentes $T_{p} M$, em todos os pontos $p \in M$.

Definição 1.1.7. Seja $M$ uma variedade diferenciável de dimensão $m$. O conjunto

$$
T M=\bigcup_{p \in M}\{p\} \times T_{p} M
$$


de todos os espaços tangente $T_{p} M$, com $p \in M$, "colados" de uma maneira natural é chamado de fibrado tangente de $M$.

Proposição 1.1.3. Dada uma variedade diferenciável $m$-dimensional $M$, o fibrado tangente $T M$ tem naturalmente a estrutura de uma variedade $2 m$-dimensional.

Informalmente, o fibrado tangente de uma variedade é obtido por considerar-se todos os espaços tangentes, e reuní-los em um conjunto diferenciável e sem sobreposição, como ilustra a Figura 1.1 onde a variedade em questão é o círculo unitário em $\mathbb{R}^{2}$.
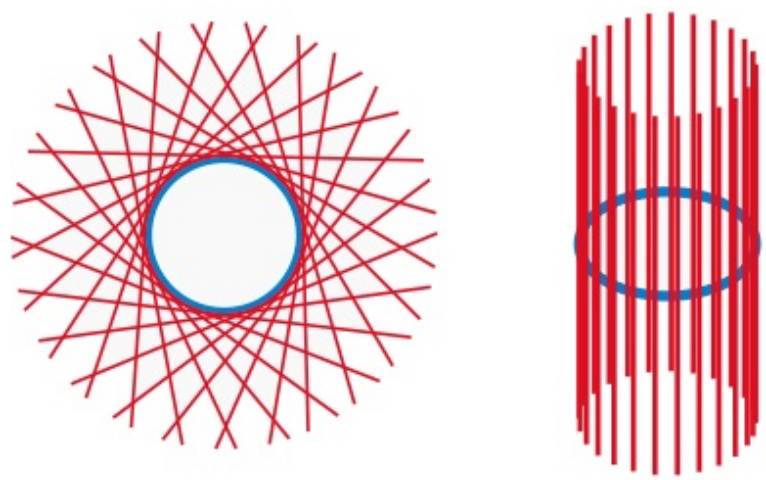

Figura 1.1: Representação do fibrado tangente de $S^{1}$

Agora, que já vimos a definição de espaço vetorial tangente, podemos definir o conceito de diferenciabilidade de uma aplicação $f: M \rightarrow N$ entre variedades diferenciáveis, utilizando nosso conhecimento prévio a cerca das aplicações deferenciáveis definidas em um subconjunto aberto de $\mathbb{R}^{m}$ com valores em $\mathbb{R}^{n}$.

Definição 1.1.8. Sejam $M, N$ variedades diferenciáveis e uma aplicação $f: M \rightarrow N$, dizemos que $f$ é diferenciável em $p \in M$ se existem cartas $\varphi: U_{1} \rightarrow V_{1}$ para $M$ com $p \in U_{1}$ e $\psi: U_{2} \rightarrow V_{2}$ para $N$ com $f\left(U_{1}\right) \subset U_{2}$ tal que $\psi \circ f \circ \varphi^{-1}$ é diferenciável em $\varphi(p)$. Diremos simplesmente que $f$ é diferenciável se ela o for em todos os pontos de $M$. E chamaremos $f$ uma aplicação de classe $C^{k}$ quando $\psi \circ f \circ \varphi^{-1}$ for de classe $C^{k}$.

Podemos provar que a definição acima não depende das escolhas das parametrizações $\varphi$ e $\psi$, sendo então coerente.

Nem sempre é fácil verificar se um espaço topológico $M$ é uma variedade diferenciável, porém, existe um importante resultado relacionado ao conceito de valor regular que nos ajuda a decidir se $M$ é uma variedade diferenciável. 


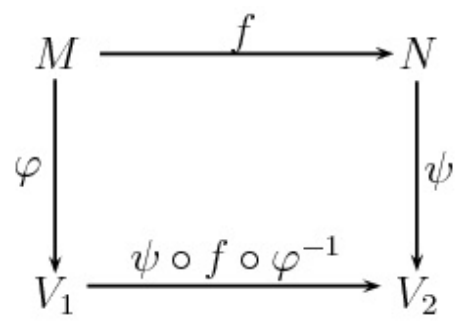

Definição 1.1.9. Sejam $M$ uma variedade diferenciável $m$-dimensional, $N$ uma variedade diferenciável $n$-dimensional e $f: M \rightarrow N$ uma aplicação diferenciável. Dizemos que:

(1) $f$ é uma imersão em $p$ se a aplicação derivada $d f_{p}$ é injetiva (portanto $n \geq m$ ) em $p \in M$. Isto é, o posto da matriz jacobiana $[J f(p)]_{n \times m}$ é igual a $m$;

(2) $f$ é uma submersão em $p$ se a aplicação derivada $d f_{p}$ é sobrejetora (portanto $m \geq n$ ) em $p \in M$. Isto é, o posto da matriz jacobiana $[J f(p)]_{n \times m}$ é igual a $n$.

Se em $p \in M$ a aplicação $f: M \rightarrow N$ for uma submersão, $p$ é dito ponto regular de $f$. Um ponto $q \in N$ é chamado valor regular de $f$ se sua imagem inversa $f^{-1}(q)$ só contém pontos regulares.

Se em $p \in M$ a aplicação $f: M \rightarrow N$ não é submersão e nem imersão, então $p$ é dito ponto singular de $f$ e $f(p)$ é o valor singular de $f$.

Teorema 1.1.4. Sejam $N$ uma variedade diferenciável $n$-dimensional, $P$ uma variedade diferenciável $p$-dimensional, $f: N \rightarrow P$ uma aplicação diferenciável e $p \in P$ um valor regular de $f$. Então, $f^{-1}(p)=M$ é uma subvariedade $(n-p)$-dimensional de $N$ e dado $q \in M$, temos $T_{q} M=\operatorname{Ker}\left(d f_{q}\right)$, onde $d f_{q}$ representa a aplicação derivada de $f$ no ponto $q$

Sejam $M, N$ variedades diferenciáveis, $f: M \rightarrow N$ uma aplicação diferenciável e um ponto $p \in N$ vimos, no Teorema 1.1.4 que uma condição suficiente para que $f^{-1}(p)$ seja uma subvariedade de $M$ é que $p$ seja um valor regular de $f$. Deste fato é natural que façamos a seguinte pergunta: dada $S \subset N$ uma subvariedade de $N$, em que condições a imagem inversa $f^{-1}(S)$ é uma subvariedade de $M$ ? Uma resposta a esta questão é dada pela noção de transversalidade. Trata-se de uma generalização natural do conceito de valor regular. 
Definição 1.1.10. Sejam $M$ uma variedade diferenciável $m$-dimensional, $N$ uma variedade diferenciável $n$-dimensional, $f: M \rightarrow N$ uma aplicação diferenciável e $P \subset N$ uma subvariedade $p$-dimensional de $N$, dizemos que $f$ é transversal a $P$ no ponto $q \in f^{-1}(P)$ quando

$$
d f_{q}\left(T_{q} M\right)+T_{f(q)} P=T_{f(q)} N
$$

ou seja, quando a imagem de $d f_{q}$ junto com o espaço tangente a $P$ em $f(q)$ geram todo $T_{f(q)} N$. Dizemos ainda que $f$ é transversal a $P$ se, para todo ponto $q \in f^{-1}(P) f$ é transversal a $P$ em $q$.

Teorema 1.1.5. Sejam $M$ uma variedade diferenciável $m$-dimensional, $N$ uma variedade diferenciável $n$-dimensional, $f: M \rightarrow N$ uma aplicação diferenciável e $P \subset N$ uma subvariedade $p$-dimensional de $N$. Se a aplicação $f$ é transversal a subvariedade $P$ então, ou bem $f^{-1}(P)=\varnothing$ ou bem $f^{-1}(P)$ é uma subvariedade $(m-n+p)$-dimensional de $M$.

Corolário 1.1.6. Seja $f: M \rightarrow N$ uma submersão, então para toda variedade $P \subset N$ ou bem $f^{-1}(P)=\varnothing$ ou bem $f^{-1}(P)$ é uma subvariedade de $M$.

\subsection{Homotopia}

Nesta seção introduzimos noções básicas sobre homotopia, procurando ilustrá-las com alguns exemplos.

Definição 1.2.1. Sejam $X$ e $Y$ espaços topológicos, dizemos que duas aplicações contínuas $f, g: X \rightarrow Y$ são homotópicas quando existe uma aplicação contínua

$$
H: X \times I \rightarrow Y, \quad \text { com } I=[0,1]
$$

tal que $H(x, 0)=f(x)$ e $H(x, 1)=g(x)$ para todo $x \in X$. Chamaremos a aplicação $H$ de homotopia entre $f$ e $g$ e escreveremos $f \simeq g$.

Exemplo 1.2.1. Sejam $E$ um espaço vetorial normado, $Y \subset E$ um espaço topológico com a topologia induzida de $E$ e $X$ um espaço topológico qualquer. Dadas aplicações contínuas 
$f, g: X \rightarrow Y$, suponhamos que, para todo $x \in X$, o segmento de reta $[f(x), g(x)]$ esteja contido em $Y$. Então, $f \simeq g$.

De fato, denotando o intervalo $[0,1]$ por $I$, basta definirmos $H(x, t)=(1-t) f(x)+t g(x)$ para obtermos uma homotopia $H: X \times Y \rightarrow Y$ entre $f$ e $g$.

Em particular, dada uma aplicação constante

$$
\begin{gathered}
f: X \quad \longrightarrow \quad E \\
x \longmapsto c
\end{gathered}
$$

toda aplicação contínua $g: X \rightarrow E$ é homotópica a $f$.

Exemplo 1.2.2. Seja $S^{n}$ a esfera unitária de $\mathbb{R}^{n+1}$, dadas duas aplicações contínuas $f, g: X \rightarrow S^{n}$, se $f(x) \neq-g(x)$ para todo $x \in X$, isto é, se $f(x)$ e $g(x)$ nunca são pontos antípodas, então $f \simeq g$.

Proposição 1.2.3. Sejam $X$ e $Y$ espaços topológicos. A relação de homotopia $f \simeq g$ é uma relação de equivalência no conjunto das aplicações contínuas de $X$ em $Y$.

Proposição 1.2.4. Sejam $X, Y$ e $Z$ espaços topológicos, consideremos aplicações $f, f^{\prime}$ : $X \rightarrow Y$ e $g, g^{\prime}: Y \rightarrow Z$ aplicações contínuas. Se $f \simeq f^{\prime}$ e $g \simeq g^{\prime}$, então $g \circ f \simeq g^{\prime} \circ f^{\prime}$. Demonstração: Seja $I=[0,1]$, denotemos por $H: X \times I \rightarrow Y$ uma homotopia entre $f$ e $f^{\prime}$ e por $K: Y \times I \rightarrow Z$ uma homotopia entre $g$ e $g^{\prime}$. Definindo $L: X \times I \rightarrow Z$ por $L(x, t)=K(H(x, t), t)$, temos que $L$ é uma homotopia entre $g \circ f$ e $g^{\prime} \circ f^{\prime}$.

Existe uma estreita relação entre homotopia e o problema de estender continuamente a todo o espaço uma aplicação contínua definida num subconjunto fechado desse espaço. Um exemplo disto pode ser visto na seguinte proposição.

Proposição 1.2.5. Sejam $X$ um espaço topológico e $\bar{B}_{1}(0)$ a bola fechada de centro 0 e raio 1 de $\mathbb{R}^{n+1}$. Uma aplicação contínua $f: S^{n} \rightarrow X$ estende-se continuamente à $\bar{B}_{1}(0)$ se, e somente se, é homotópica a uma constante.

Demonstração: Denotemos por $I$ o intervalo $[0,1]$ e consideremos a aplicação

$$
\begin{aligned}
\varphi: S^{n} \times I & \longrightarrow \bar{B}_{1}(0) \\
(x, t) & \longmapsto(1-t) x
\end{aligned}
$$


que é contínua, sobrejetora e igual a 0 em $S^{n} \times 1$. Se $\tilde{f}: \bar{B}_{1}(0) \rightarrow X$ é uma extensão contínua de $f: S^{n} \rightarrow X$, então

$$
H=\tilde{f} \circ \varphi: S^{n} \times I \rightarrow X
$$

é uma homotopia entre $f$ e a aplicação constante $g: S^{n} \rightarrow X$, definida por $g(x)=\tilde{f}(0)$. Reciprocamente, suponhamos que $H: S^{n} \rightarrow X$ seja uma homotopia entre $f$ e uma aplicação constante $g: S^{n} \rightarrow X$, dada por $g(x)=p$, para todo $x \in S^{n}$. Seja a aplicação $\tilde{f}: S^{n} \rightarrow X$ definida da seguinte maneira,

$$
\tilde{f}(x)=\left\{\begin{array}{l}
H\left(\frac{x}{|x|}, 1-|x|\right), \text { se } x \neq 0 \\
p, \text { se } x=0
\end{array}\right.
$$

Assim, $\tilde{f}$ é contínua e $\left.\tilde{f}\right|_{S^{n}}=f$.

Definição 1.2.2. Sejam $X$ e $Y$ espaços topológicos, dizemos que uma aplicação contínua $f: X \rightarrow Y$ é uma equivalência homotópica quando existe uma aplicação contínua $g$ : $Y \rightarrow X$ tal que $g \circ f \simeq i d: X \rightarrow X$ e $f \circ g \simeq i d: Y \rightarrow Y$. Neste caso, $g$ chama-se uma equivalência inversa de $f$ e os espaços $X$ e $Y$ dizem-se ter o mesmo tipo de homotopia.

Exemplo 1.2.6. Dado um ponto $a \in \mathbb{R}^{n}$, seja $\bar{B}_{\varepsilon}(a)$ a bola fechada de centro a e raio $\varepsilon>0$ em $\mathbb{R}^{n}$. Então, $\bar{B}_{\varepsilon}(a) \backslash\{a\}$ e a esfera unitária $S^{n-1}$ têm o mesmo tipo de homotopia.

De fato, sejam

$$
\rho=\rho_{a, \varepsilon}: \bar{B}_{\varepsilon}(a) \backslash\{a\} \rightarrow S^{n-1} \quad \text { e } \quad j=j_{a, \varepsilon}: S^{n-1} \rightarrow \bar{B}_{\varepsilon}(a) \backslash\{a\}
$$

as aplicações definidas por

$$
\rho(x)=\frac{x-a}{\|x-a\|} \text { e } j(y)=a+\varepsilon y
$$


assim, $\rho \circ j=i d: S^{n-1} \rightarrow S^{n-1}$. Além disso, considerando a aplicação contínua $F: \bar{B}_{\varepsilon}(a) \backslash\{a\} \times I \rightarrow \bar{B}_{\varepsilon}(a) \backslash\{a\}$ dada por

$$
F(x, t)=(1-t)\left(a+\varepsilon \frac{x-a}{\|x-a\|}\right)+t x .
$$

temos $j \circ \rho \simeq i d: \bar{B}_{\varepsilon}(a) \backslash\{a\} \rightarrow \bar{B}_{\varepsilon}(a) \backslash\{a\}$.

Fazendo $\varepsilon=\infty$ no Exemplo 1.2.6. obtemos $B=\mathbb{R}^{n}$. Continuamos tendo $\mathbb{R}^{n} \backslash\{a\}$ e $S^{n-1}$ do mesmo tipo de homotopia, a equivalência homotópica

$$
\rho=\rho_{a, \infty}: \mathbb{R}^{n} \backslash\{a\} \rightarrow S^{n-1}
$$

define-se do mesmo modo, mas sua inversa $j=j_{a, \infty}: S^{n-1} \rightarrow \mathbb{R}^{n} \backslash\{a\}$ é definida por $j(y)=a+y$.

Definição 1.2.3. Um espaço topológico $X$ é dito contrátil quando ele tem o mesmo tipo de homotopia de um ponto.

\subsection{Homotopia de pares e homotopia relativa}

Definição 1.3.1. Diremos que $(X, A)$ é um par de espaços topológicos quando $A$ for um subespaço de $X$. Dados os pares $(X, A)$ e $(Y, B)$, uma aplicação contínua $f:(X, A) \longrightarrow$ $(Y, B)$ é uma aplicação contínua $f: X \longrightarrow Y$ tal que $f(A) \subset B$.

Definição 1.3.2. Dadas as aplicações contínuas $f, g:(X, A) \longrightarrow(Y, B)$, uma homotopia de pares entre $f$ e $g$ é uma aplicação contínua

$$
H:(X \times I, A \times I) \longrightarrow(Y, B)
$$

tal que $H(x, 0)=f(x)$ e $H(x, 1)=g(x)$, para todo $x \in X$. Deve-se portanto ter $H_{t}(A) \subset B, \forall t \in I$.

Observação 1.3.1. O caso em que $B$ se reduz a um ponto $y_{0}$ é frequentemente usado, este ponto é chamado de ponto básico do espaço $Y$. Neste caso, durante uma homotopia entre 
duas aplicações $f, g:(X, A) \longrightarrow\left(Y, y_{0}\right)$, a aplicação $H_{t}$ deve ser constante no subespaço A.

Definição 1.3.3. Dadas as aplicações contínuas $f, g: X \longrightarrow Y$. Diremos que $f$ é homotópica a $g$ relativamente a um subespaço $A \subset X$, e escreve-se

$$
f \simeq g(\operatorname{rel} . A)
$$

quando existe uma homotopia $H$ entre $f$ e $g$ tal que $H(x, t)=f(x)=g(x)$ para todo $x \in A$.

\subsection{Grupos de Homotopia}

\subsubsection{O Grupo Fundamental}

Uma referência para este assunto é [17].

Definição 1.4.1. Um caminho em um espaço topológico $X$ é uma aplicação contínua $a: J \longrightarrow X$, em que $J$ é um intervalo compacto $J=\left[s_{0}, s_{1}\right]$. Vamos trabalhar sempre com caminhos definidos no intervalo $I=[0,1]$.

Definição 1.4.2. Diremos que $a, b: I \longrightarrow X$ são caminhos homotópicos quando tivermos $a \simeq b($ rel. $\partial I)$.

Definição 1.4.3. Sejam $a, b: I \longrightarrow X$ caminhos tais que $a(1)=b(0)$. Definimos o produto $a b$ como sendo:

$$
a b(s)=\left\{\begin{array}{cc}
a(2 s), & \text { se } 0 \leq s \leq 1 / 2 \\
b(2 s-1), & \text { se } 1 / 2 \leq s \leq 1
\end{array}\right.
$$

Proposição 1.4.1. Sejam $a, b, c: I \longrightarrow X$ caminhos tais que $a(1)=b(0)$ e $b(1)=c(0)$. Sejam $\alpha=[a], \beta=[b]$ e $\gamma=[c]$ suas respectivas classes de homotopia, $x=a(0)$, $y=a(1), e_{x}, e_{y}$ os caminhos constantes sobre estes pontos e $\varepsilon_{x}=\left[e_{x}\right], \varepsilon_{y}=\left[e_{y}\right]$ as classes de homotopia destes caminhos. Então: 
1. $\alpha \alpha^{-1}=\varepsilon_{x}$;

2. $\alpha^{-1} \alpha=\varepsilon_{y}$;

3. $\varepsilon_{x} \alpha=\alpha=\alpha \varepsilon_{y}$;

4. $(\alpha \beta) \gamma=\alpha(\beta \gamma)$;

Assim, o conjunto das classes de homotopia (com extremos fixos) dos caminhos em um espaço topológico $X$, munido da lei de composição dada na Definição 1.4.3 chama-se grupóide fundamental de $X$.

Definição 1.4.4. O subconjunto $\pi_{1}\left(X, x_{0}\right)$ do grupóide fundamental formado pelas classes de homotopia de caminhos fechados com ponto base em $x_{0}$ constitui um grupo, chamado grupo fundamental de $X$, com base no ponto $x_{0}$. O elemento neutro deste grupo é a classe de homotopia $\varepsilon=\varepsilon_{0}$ do caminho constante no ponto $x_{0}$.

Observação 1.4.2 (Grupos de Homotopia de ordem n). Um tema importantíssimo para o desenvolvimento do trabalho apresentado ao longo desta dissertação é o de grupos de homotopia de ordem n. A definição do n-ésimo grupo de homotopia de um espaço, $\pi_{n}\left(X, x_{0}\right)$, é estritamente análoga à definição de grupo fundamental. Nós substituímos o intervalo $I=[0,1]$ pelo $n$-cubo $I^{n}$ consistindo dos pontos $t=\left(t_{1}, \ldots, t_{n}\right)$ em $\mathbb{R}^{n}$ tais que $0 \leq t_{i} \leq 1$ com $i=1, \ldots, n$. Uma ótima referência para este assunto é [26].

O seguinte conceito de $q$-conexidade será fundamental para nossos estudos no Capítulo 5 .

Definição 1.4.5. (veja [26]) Diremos que um espaço $X$ é q-conexo $(q \geq 0)$ se ele for conexo por caminhos e $\pi_{i}(X)=0$ para $i=1, \ldots, q$.

Definição 1.4.6. Diremos que um par de espaços topológicos $(X, Y)$ é q-conexo $(q \geq 0)$ se ele for conexo por caminhos e $\pi_{i}(X, Y)=0$ para $i=1, \ldots, q$.

\subsection{Homologia singular}

Há diversas maneiras de tratar-se a teoria de homologia sobre espaços topológicos. Em particular, as homologias singular e simplicial que coincidem em espaços triangularizáveis 
e variedades compactas. Como ao longo desta exposição trabalharemos com complexos $C W$, que são triangulárizáveis, optamos aqui por estudar a abordagem da homologia singular. Faremos o estudo dos R-módulos de homologia singular com coeficientes em um anel $\mathrm{R}$ comutativo com identidade. Uma referência para este assunto é [21].

\section{Simplexos em $\mathbb{R}^{n}$}

Definição 1.5.1. Dados $(p+1)$ pontos em $\mathbb{R}^{n}, p \leq n$, dizemos que $\left\{X_{0}, \ldots, X_{p}\right\}$ é uma coleção geometricamente independente se os vetores $\overrightarrow{v_{1}}=X_{1}-X_{0}, \overrightarrow{v_{2}}=X_{2}-X_{0}, \ldots$, $\overrightarrow{v_{p}}=X_{p}-X_{0}$ são linearmente independentes.

Definição 1.5.2. Seja $\left\{X_{0}, \ldots, X_{p}\right\} \subset \mathbb{R}^{n}$ uma coleção geometricamente independente de $\mathbb{R}^{n}, p \leq n$. O $p$-simplexo $S$ gerado por $X_{0}, \ldots, X_{p}$ é a envoltória convexa:

$$
S=E C\left(\left\{X_{0}, \ldots, X_{p}\right\}\right)
$$

Os pontos $X_{i}$ são chamados vértices do $p$-simplexo $\mathrm{S}$.

Teorema 1.5.1. (Teorema das coordenadas baricêntricas) Sejam $\left\{X_{0}, \ldots, X_{p}\right\}$ uma coleção de $(p+1)$ pontos geometricamente independentes em $\mathbb{R}^{n}$, com $p \leq n$. Considere o seguinte conjunto:

$$
A_{p}=\left\{t_{0} X_{0}+\ldots+t_{p} X_{p} ; \quad 0 \leq t \leq 1 \text { e } \sum_{i=0}^{p} t_{i}=1\right\} \subset \mathbb{R}^{n}
$$

(i) $A_{p}=E C\left(\left\{X_{0}, \ldots, X_{p}\right\}\right)=p$-simplexo gerado por $X_{0}, \ldots, X_{p}$;

(ii) Se $\sum_{i=0}^{p} t_{i} X_{i}=\sum_{i=0}^{p} s_{i} X_{i}, \quad 0 \leq t_{i}, s_{i} \leq 1 \mathrm{com} \sum_{i=0}^{p} t_{i}=\sum_{i=0}^{p} s_{i}=1$, então $t_{i}=s_{i}$ para todo $i=1, \ldots, p$, ou seja: todo elemento de $A_{p}$ se escreve de maneira única como combinação linear dos pontos $\left\{X_{0}, \ldots, X_{p}\right\}$.

Observação 1.5.2. Concluímos que escolhida uma ordenação $\left\{X_{0}, \ldots, X_{p}\right\}$ para $(p+1)$ pontos geometricamente independentes em $\mathbb{R}^{n}$, com $p \leq n$, cada ponto $X \in E C\left(\left\{X_{0}, \ldots, X_{p}\right\}\right)$ é univocamente determinado por uma $(p+1)$-upla $\left(t_{0}, t_{1}, \ldots, t_{p}\right)$, com $0 \leq t_{i} \leq 1$ e $\sum_{i=0}^{p} t_{i}=1$ denominada as coordenadas baricêntricas do ponto $X$. 
Definição 1.5.3. Sejam $X_{0}=e_{1}=(1,0, \ldots, 0), X_{1}=e_{2}=(0,1,0, \ldots, 0), \ldots, X_{p}=$ $e_{p+1}=(0,0, \ldots, 0,1)$. O $p$-simplexo $E C\left(\left\{X_{0}, \ldots, X_{p}\right\}\right)$, nesse caso, é denominado $p$-simplexo padrão e será denotado por $\Delta_{p} \in \mathbb{R}^{p+1}$

\section{Homologia singular de um espaço topológico $X$ com coeficientes em um anel $R$}

Fixemos $R$ um anel comutativo com a identidade $1_{R}$ e consideremos $\Delta_{p} \subset \mathbb{R}^{p+1} \mathrm{o}$ $p$-simplexo padrão munido da topologia induzida de subespaço em $\mathbb{R}^{p+1}$ (munido da topologia usual).

Definição 1.5.4. Para cada espaço topológico $X$, um $p$-simplexo singular em $X$ é uma função contínua $\sigma: \Delta_{p} \rightarrow X$.

Denotaremos por

$$
C_{p}(X)=\left\{\sigma: \Delta_{p} \rightarrow X ; \quad \sigma \text { é contínua }\right\}
$$

o conjunto de todos os $p$-simplexos singulares de $X$.

Definição 1.5.5. Para cada $p \geq 0$, definimos o $R$-módulo livre cuja base é o conjunto de todos os $p$-simplexos singulares de $X$ :

$$
S_{p}\left(C_{p}(X), R\right)=\left\{f: C_{p}(X) \rightarrow R ; f(\sigma) \neq 0, \text { apenas para um n finito de elementos } \sigma\right\}
$$

Um elemento típico de $S_{p}\left(C_{p}(X), R\right)$ é uma combinação linear formal $\alpha_{1} \sigma_{1}+\ldots+\alpha_{r} \sigma_{r}$, onde $\alpha_{i} \in R$ e $\sigma_{i}: \Delta_{p} \rightarrow X$ é um $p$-simplexo singular de $X$.

$$
\begin{aligned}
\sum_{i=0}^{r} \alpha_{i} \sigma_{i} \longleftrightarrow f: C_{p}(X) & \rightarrow R \\
\sigma & \mapsto f(\sigma)= \begin{cases}\alpha_{i} ; & \text { se } \sigma=\sigma_{i} i=1, \ldots, r \\
0, & \text { se } \sigma \neq \sigma_{i}\end{cases}
\end{aligned}
$$

Os elementos de $S_{p}\left(C_{p}(X) ; R\right)$ são chamados $\mathbf{p}$-cadeias singulares de $\mathbf{X}$ com coeficientes em $\mathbf{R}$. 
A seguir, definiremos o complexo de cadeias $\left\{S_{p}(X ; R) ; \partial\right\}_{p \geq 0}$. Para isso, convencionaremos que $\left\{S_{p}(X ; R)\right\}=\{0\}$ para todo $p<0$.

\section{Os operadores face}

Definição 1.5.6. Seja $\sigma: \Delta_{p} \rightarrow X$ um $p$-simplexo singular de $X$. Considere a inclusão

$$
\begin{array}{ccc}
\Delta_{p-1} & \stackrel{\varepsilon_{i}}{\hookrightarrow} & \Delta_{p} \\
\left(t_{0}, \ldots, t_{i}, \ldots, t_{p-1}\right) & \hookrightarrow & \left(t_{0}, \ldots, t_{i-1}, 0, t_{i}, \ldots, t_{p-1}\right),
\end{array}
$$

que insere o zero na i-ésima posição. Para cada $i=1, \ldots, p$ definimos a i-ésima face de $\sigma$ como sendo o $(p-1)$-simplexo singular de $X$ dado pela composição

$$
\sigma \circ \varepsilon_{i}: \Delta_{p-1} \rightarrow X
$$

que é contínua e denotada por $\partial_{i} \sigma: \Delta_{p-1} \rightarrow X$.

Assim, para cada $i=1, \ldots, p$ e para cada $p$-simplexo singular $\sigma \in C_{p}(X)$, associamos a sua i-ésima face $\partial_{i} \sigma \in C_{p-1}(X)$. Isso define uma função

$$
\begin{array}{cccc}
\partial_{i}: C_{p}(X) & \longrightarrow & C_{p-1}(X) \subset S_{p-1}(X ; R) \\
\sigma & \longmapsto & \partial_{i} \sigma
\end{array}
$$

para todo $i=1, \ldots, p$, e desde que $C_{p}(X)$ é base para o R-módulo livre $S_{p}(X ; R), \partial_{i}$ se estende por linearidade a um único R-homomorfismo $\partial_{i}: S_{p}(X ; R) \rightarrow S_{p-1}(X ; R)$, chamado operador face. O R-homomorfismo $\partial: S_{p}(X ; R) \rightarrow S_{p-1}(X ; R)$ dado pela soma alternada dos operadores face

$$
\partial=\partial_{0}-\partial_{1}+\partial_{2}-\ldots+(-1)^{p} \partial_{p}=\sum_{i=0}^{p}(-1)^{i} \partial_{i}
$$

é chamado operador bordo.

Teorema 1.5.3. O operador $\partial: S_{p}(X ; R) \rightarrow S_{p-1}(X ; R)$, definido por $\sum_{i=0}^{p}(-1)^{i} \partial_{i}$, é tal que $\partial \circ \partial=0$. 
Definição 1.5.7. Fixado um anel comutativo com identidade $R$ para cada espaço topológico $X$, criamos um complexo de cadeias $S_{*}(X ; R)=\left\{S_{p}(X ; R), \partial\right\}_{p \geq 0}$, ou seja,

$$
S_{*}(X ; R): \cdots \stackrel{\partial}{\longrightarrow} S_{p}(X ; R) \stackrel{\partial}{\longrightarrow} S_{p-1}(X ; R) \stackrel{\partial}{\longrightarrow} \cdots \stackrel{\partial}{\longrightarrow} S_{1}(X ; R) \stackrel{\partial}{\longrightarrow} S_{0}(X ; R) \stackrel{\partial}{\longrightarrow} 0,
$$

usaremos as seguintes terminologias:

$Z_{p}\left(S_{*}(X ; R)\right) \doteq Z_{p}(X ; R)=\operatorname{Ker}(\partial)$ o submódulo dos p-ciclos,

$B_{p}\left(S_{*}(X ; R)\right) \doteq B_{p}(X ; R)=\operatorname{Im}(\partial)$ o submódulo dos p-bordos e

$H_{p}\left(S_{*}(X ; R)\right) \doteq H_{p}(X ; R)=\frac{Z_{p}(X ; R)}{B_{p}(X ; R)}$ o p-ésimo R-módulo de homologia de $X$ com coeficientes em R.

Um elemento típico de $H_{p}(X ; R)$ é da forma

$$
\mu=z_{p}+B_{p}(X ; R)
$$

onde $z_{p} \in Z_{p}(X ; R)$ é um p-ciclo; $\mu$ é chamada classe de homologia de X com coeficientes em $\mathbf{R}$, representada pelo p-ciclo $z_{p}$.

\section{Construção do R-homomorfismo induzido em homologia}

Dada uma função contínua $f: X \rightarrow Y$, queremos definir um $R$-homomorfismo $f_{*}: H_{p}(X ; R) \rightarrow H_{p}(Y ; R)$ para cada $p \geq 0$. Para isso, precisamos definir uma aplicação de cadeias

$$
f_{\#}: S_{p}(X ; R) \rightarrow S_{p}(Y ; R)
$$

para cada $p \geq 0$, entre os complexos de cadeias $S_{*}(X ; R)$ e $S_{*}(Y ; R)$.

Observemos que, dado um $p$-simplexo singular de $X, \sigma: \Delta_{p} \rightarrow X$, a composição

$$
f \circ \sigma: \Delta_{p} \stackrel{\sigma}{\longrightarrow} X \stackrel{f}{\longrightarrow} Y
$$


é um $p$-simplexo singular de $Y$ e determina uma função

$$
\begin{aligned}
C_{p}(X) & \longrightarrow C_{p}(Y) \quad \subset S_{p}(Y ; R) \\
\sigma & \longmapsto f \circ \sigma
\end{aligned}
$$

para cada $p \geq 0$. Desde que $C_{p}(X)$ é base para o $R$-módulo livre $S_{p}(X ; R)$ e $C_{p}(Y) \subset S_{p}(Y ; R)$ tal função se estende por linearidade a um único $R$-homomorfismo $f_{\#}: S_{p}(X ; R) \rightarrow S_{p}(Y ; R)$, definido por

$$
f_{\#}\left(\alpha_{1} \sigma_{1}+\ldots+\alpha_{r} \sigma_{r}\right)=\alpha_{1}\left(f \circ \sigma_{1}\right)+\ldots+\alpha_{r}\left(f \circ \sigma_{r}\right)
$$

Teorema 1.5.4. O $R$-homomorfismo $f_{\#}: S_{p}(X ; R) \rightarrow S_{p}(Y ; R)$ é uma aplicação de cadeias.

Portanto, $f_{\#}: S_{p}(X ; R) \rightarrow S_{p}(Y ; R)$ induz um bem definido $R$-homomorfismo $f_{*}: H_{p}(X ; R) \rightarrow H_{p}(Y ; R)$, dado por

$$
f_{*}\left(z_{p}\right)+B_{p}(X ; R)=f_{\#}\left(z_{p}\right)+B_{p}(Y ; R)
$$

onde

$$
f_{\#}\left(z_{p}\right)=f_{\#}\left(\alpha_{1} \sigma_{1}+\ldots+\alpha_{r} \sigma_{r}\right)=\alpha_{1}\left(f \circ \sigma_{1}\right)+\ldots+\alpha_{r}\left(f \circ \sigma_{r}\right) .
$$

O seguinte resultado será fundamental para nossos estudos no Capítulo 4, como referência para este assunto sugerimos [10].

Teorema 1.5.5 (Teorema de Hurewicz). Se um par $(X, A)$ for $(n-1)$-conexo, $n \geq 2$, com $A$ simplesmente conexo e não vazio, então $H_{i}(X, A)=0$ para $i<n$ e $\pi_{n}(X, A) \cong$ $H_{n}(X, A)$.

\subsection{Complexos $C W$}

De maneira informal, um complexo $C W$ é construído a partir de blocos básicos denominados células. A definição precisa descreve como as células podem ser "topológicamente 
coladas". Uma célula aberta $n$-dimensional é um espaço topológico homeomorfo a uma bola aberta $n$-dimensional.

Um complexo $C W$ é um espaço topológico de Hausdorff $X$ junto com uma partição de $X$ em células abertas (de várias dimensões) tais que: Para cada célula aberta $n$ dimensional $C$ em $X$, existe uma aplicação contínua $f$ da bola aberta $n$-dimensional em $X$, que aplica esta bola aberta homeomorficamente sobre $C$ e a imagem do bordo da bola aberta, isto é, a imagem do bordo da célula aberta $C$, intersecta apenas um número finito de outras células. Um conjunto de $X$ é fechado (em $X)$ se, e somente se, ele intersecta o fecho de cada célula segundo um conjunto fechado.

Indicamos como referências para este assunto [3] e [24].

\subsection{Fibrações, Fibrados e Fibrados Vetoriais}

Apresentamos nesta seção conceitos básicos sobre fibrações, fibrados e fibrados vetoriais. Para um estudo mais completo sugerimos [26].

Definição 1.7.1 (Fibrado). Sejam E, B, F espaços topológicos. Um fibrado $\xi$ sobre B, com fibra $F$ é uma terna $\xi=(E, p, F)$ satisfazendo as condições:

1. $p: E \rightarrow B$ é uma aplicação contínua e sobrejetora chamada projeção;

2. para cada $b \in B$, existem um aberto $U_{b} \subset B, b \in U_{b}$, e um homeomorfismo $\phi_{b}$, $\phi_{b}: U_{b} \times F \longrightarrow p^{-1}\left(U_{b}\right)$ tal que $p \circ \phi_{b}=\Pi_{1}$, em que $\Pi_{1}$ é a projeção na primeira coordenada (condição de trivialidade local).

Neste caso, denominamos $E$ o espaço total do fibrado e $B$ o espaço base. Note que, para cada $b \in B$, a fibra $p^{-1}(b)$ é homeomorfa a $F$.

Definição 1.7.2 (Fibrado diferenciável). Sejam E, B, F variedades diferenciáveis. Um fibrado diferenciável $\xi$ sobre $B$, com fibra $F$ é uma terna $\xi=(E, p, F)$ satisfazendo as condições:

1. $p: E \rightarrow B$ é uma submersão sobrejetora; 
2. para cada $b \in B$, existem um aberto $U_{b} \subset B, b \in U_{b}$, e um difeomorfismo $\phi_{b}$, $\phi_{b}: U_{b} \times F \longrightarrow p^{-1}\left(U_{b}\right)$ tal que $p \circ \phi_{b}=\Pi_{1}$, em que $\Pi_{1}$ é a projeção na primeira coordenada (condição de trivialidade local).

Neste caso, para cada $b \in B$, a fibra $p^{-1}(b)$ é difeomorfa a $F$.

Definição 1.7.3. Um fibrado vetorial (real ou complexo) $\xi$ sobre B é uma terna, formada por:

1. Um espaço topológico $E=E(\xi)$ chamado espaço total,

2. uma aplicação (contínua) $\pi: E \rightarrow B$ sobrejetora chamada projeção,

3. para cada $b \in B, \pi^{-1}(b)$ tem uma estrutura de espaço vetorial sobre $\mathbb{K}(\mathbb{K}=\mathbb{R}$ ou C).

E deve satisfazer à seguinte condição:

Condição de trivialidade local: Para cada ponto $b \in B$ deve existir uma vizinhança $U \subset B$, um inteiro $n \geq 0$ e um homeomorfismo

$$
h: U \times \mathbb{K}^{n} \rightarrow \pi^{-1}(U)
$$

tal que, para cada $b \in U$, a correspondência $x \mapsto h(b, x)$ define um isomorfismo entre o espaço vetorial $\mathbb{K}^{n}$ e o espaço vetorial $\pi^{-1}(b)$.

O par $(U, h)$ será chamado sistema de coordenadas local para $\xi$ em b. Se pudermos escolher $U$ igual ao espaço base, então $\xi$ será chamado de fibrado trivial.

O espaço vetorial $\pi^{-1}(b)$ é chamado de fibra sobre $b$, e pode ser denotado por $F_{b}$ ou por $F_{b}(\xi)$. Observemos que $F_{b}$ nunca é vazio, entretanto pode ser formado por apenas um ponto.

O conceito de fibrado vetorial diferenciável pode ser definido pedindo apenas que $B$ e $E$ sejam variedades diferenciáveis, que $\pi$ seja uma aplicação suave e que para cada $b \in B$ exista um sistema de coordenadas locais $(U, h)$ com $b \in U$ tal que $h$ seja um difeomorfismo.

Consideremos agora dois fibrados vetoriais $\xi$ e $\nu$ sobre o mesmo espaço base $B$. 
Definição 1.7.4. Dizemos que $\xi$ é isomorfo à $\nu$, escrevemos $\xi \cong \nu$, se existir um homeomorfismo

$$
f: E(\xi) \rightarrow E(\nu)
$$

entre os espaços totais que aplique cada espaço vetorial $F_{b}(\xi)$ isomorficamente sobre o espaço vetorial correspondente $F_{b}(\nu)$.

Definição 1.7.5. Uma seção de um fibrado vetorial $\xi$ com base $B$ é uma função contínua

$$
s: B \rightarrow E(\xi)
$$

a qual leva $b$ dentro de sua fibra correspondente $F_{b}(\xi)$. Uma seção do fibrado tangente à uma variedade suave $M$ é usualmente chamada de campo de vetores de $M$.

Definição 1.7.6. Uma fibração é uma generalização da noção de fibrado. O conceito de fibração é como o de um fibrado, exceto pelo fato que as fibras não precisam ser homeomorfas, ao contrário, é pedido apenas que elas tenhas o mesmo tipo de homotopia.

Observação 1.7.1. É importante observar que ao longo de nossa exposição sobre o Teorema da Fibração de Milnor provaremos que uma aplicação $\phi$ é um fibrado diferenciável localmente trivial, embora o nome do teorema mencione a palavra "fibração". Esta denominação ocorre pois um dos fatores mais importantes deste resultado é a demonstração de que as fibras desta fibração (fibras de Milnor) tem o tipo de homotopia de um buquê de esferas.

\subsection{Teoria de singularidades}

Nesta seção, apresentamos as definições e principais resultados da teoria de singularidades que serão usados para o desenvolvimento deste trabalho. Para o leitor interessado recomendamos [1, 19.

Um dos nossos objetivos é estudar localmente funções analíticas $f: \mathbb{C}^{n+k} \rightarrow \mathbb{C}^{k}$. Para isso, introduzimos uma relação de equivalência no espaço das funções analíticas definidas num aberto contendo a origem de $\mathbb{C}^{n}$, da seguinte forma: 
Definição 1.8.1. Dizemos que a função $f: \mathbb{C}^{n} \rightarrow \mathbb{C}$ é equivalente à função $g: \mathbb{C}^{n} \rightarrow \mathbb{C}$, se existe um aberto $U \subset \mathbb{C}^{n}$, com $0 \in U$, tal que $f_{\mid U} \equiv g_{\mid U}$. As classes de equivalência são chamadas de germes, denotadas por $f:\left(\mathbb{C}^{n}, 0\right) \rightarrow \mathbb{C}$, ou simplesmente, por $f$. A coleção de todos esses germes de funções citados acima é denotada por $\mathcal{O}_{n}$. Observemos que $\mathcal{O}_{n}$ é um anel noetheriano local cujo ideal maximal é dado por $\mathcal{M}_{n}=\left\{f \in \mathcal{O}_{n}: f(0)=0\right\}$.

Definição 1.8.2. Denotamos por $\mathcal{O}_{n, p}$ o conjunto dos germes de aplicações analíticas $f:\left(\mathbb{C}^{n}, 0\right) \rightarrow \mathbb{C}^{p}$ e por $\mathcal{O}_{n, p}^{0}$ o conjunto dos germes $f:\left(\mathbb{C}^{n}, 0\right) \rightarrow\left(\mathbb{C}^{p}, 0\right)$.

Proposição 1.8.1. $\mathcal{O}_{n, p}$ é um $\mathcal{O}_{n}$-módulo livre de posto $p$.

\subsection{Variedades algébricas e espaços analíticos complexos}

Nesta seção trabalharemos com o caso complexo.

\section{Variedades algébricas}

O $n$-espaço afim sobre $\mathbb{C}$, denotado por $\mathbb{A}^{n}$ é o conjunto de todas as $n$-uplas de elementos de $\mathbb{C}$. Um ponto $p \in \mathbb{A}^{n}$ é dado por $p=\left(a_{1}, \ldots, a_{n}\right)$, com $a_{i} \in \mathbb{C}$, e $a_{i}$ a $i$-ésima coordenada de $p$.

Seja $\mathbb{C}\left[x_{1}, \cdots, x_{n}\right]$ o anel de polinômios em $n$ variáveis sobre $\mathbb{C}$. Podemos interpretar os elementos de $\mathbb{C}\left[x_{1}, \cdots, x_{n}\right]$ como funções do espaço afim $\mathbb{A}^{n}$ sobre $\mathbb{C}$, com $f(p)=$ $f\left(a_{1}, \ldots, a_{n}\right)$, onde $f \in \mathbb{C}\left[x_{1}, \cdots, x_{n}\right]$ e $p \in \mathbb{A}^{n}$. Desta forma, faz sentido falar no conjunto de zeros de $f$, que denotaremos por $Z(f)=\left\{p \in \mathbb{A}^{n} ; f(p)=0\right\}$. De uma forma mais geral, se $T$ for um subconjunto de $\mathbb{C}\left[x_{1}, \cdots, x_{n}\right]$, podemos definir o conjunto de zeros de $T$ como sendo o conjunto de zeros comuns a todos os elementos de $T$. Denotaremos esse conjunto da seguinte forma,

$$
Z(T)=\left\{p \in \mathbb{A}^{n} ; f(p)=0 \forall f \in T\right\}
$$

Claramente se $I$ for o ideal de $\mathbb{C}\left[x_{1}, \cdots, x_{n}\right]$ gerado por $T$ então $Z(T)=Z(I)$. Mais ainda, como $\mathbb{C}\left[x_{1}, \cdots, x_{n}\right]$ é um anel Noetheriano, qualquer ideal $I$ tem um número finito 
de geradores $f_{1}, \ldots, f_{r}, \operatorname{logo} Z(T)$ pode se expressar como o conjunto de zeros comuns de um número finito de polinômios $f_{1}, \ldots, f_{r}$.

Definição 1.9.1. Um subconjunto $Y$ de $\mathbb{A}^{n}$ é chamado conjunto algébrico se existe um subconjunto $T \subset \mathbb{C}\left[x_{1}, \cdots, x_{n}\right]$ tal que $Y=Z(T)$.

Proposição 1.9.1. A união de dois conjuntos algébricos é um conjunto algébrico. A intersecção de uma família arbitrária de conjuntos algébricos também é um conjunto algébrico.

Para a demonstração deste resultado e referência básica para o assunto ver [9].

Definição 1.9.2. Os conjuntos algébricos são os conjuntos fechados de uma topologia de $\mathbb{A}^{n}$, denominada topologia de Zariski.

Definição 1.9.3. Dizemos que $Y \subset X$, um subconjunto não vazio $Y$ contido em $X$ é irredutível se o mesmo não pode se expressar como uma união $Y=Y_{1} \cup Y_{2}$, onde $Y_{1}, Y_{2}$ são fechados em $Y$.

Definição 1.9.4. Uma variedade algébrica afim é um subconjunto irredutível de $\mathbb{A}^{n}$ (com a topologia induzida).

\section{Espaços analíticos complexos}

Analogamente ao caso de polinômios, podemos também estudar o conjunto de zeros de uma ou mais funções analíticas. Estes são os chamados espaços analíticos.

Primeiramente consideremos a seguinte definição:

Definição 1.9.5. Consideremos o conjunto de pares $\left(V_{\alpha}, U_{\alpha}\right)$, onde $U_{\alpha}$ é uma vizinhança aberta da origem em $\mathbb{C}^{n}$ e $V_{\alpha}$ subconjuntos de $U_{\alpha}$. Dois tais pares $\left(V_{1}, U_{1}\right)$ e $\left(V_{2}, U_{2}\right)$ são equivalentes se existe uma vizinhança $W \subset U_{1} \cap U_{2}$ da origem tal que $V_{1} \cap W=V_{2} \cap W$. Uma classe de equivalência destes pares é chamada germe na origem em $\mathbb{C}^{n}$.

Se $f \in \mathcal{O}_{n}$, a classe de equivalência do conjunto $\{x: f(x)=0\}$, onde $f$ é um representante do germe $f$, é denotada por $\mathcal{V}(f)$; se $f_{1}$ e $f_{2}$ são dois representantes de um mesmo germe, então os conjuntos $\mathcal{V}\left(f_{1}\right)$ e $\mathcal{V}\left(f_{2}\right)$ são iguais. 
Definição 1.9.6. Um germe de espaço analítico $(V, 0)$ em torno da origem é o germe do subconjunto

$$
V=\mathcal{V}\left(f_{1}\right) \cap \ldots \cap \mathcal{V}\left(f_{r}\right)=\mathcal{V}\left(f_{1}, \ldots, f_{r}\right),
$$

para $f_{1}, \ldots, f_{r} \in \mathcal{O}_{n}$.

Definimos o ideal de um germe de espaço analítico $V$ por

$$
\mathcal{I}(V)=\left\{f \in \mathcal{O}_{n}: V \subset f^{-1}(0)\right\} .
$$

Dizemos que um germe de espaço analítico $V$ é irredutível quando para quaisquer germes $V_{1}$ e $V_{2}$ tais que $V=V_{1} \cup V_{2}$ então $V=V_{1}$ ou $V=V_{2}$, neste caso dizemos que $V$ é uma variedade analítica.

O nosso objetivo é estudar a natureza de tais espaços analíticos na vizinhança de algum ponto fixado em $\mathbb{C}^{n}$, o qual sem perda de generalidade consideraremos a origem. Para isso precisamos definir formalmente o conceito de germe de um espaço analítico complexo (veja [6]).

Proposição 1.9.2. Seja $V$ um germe de espaço analítico, então existem um inteiro positivo $p$ e $V_{1}, \ldots, V_{p}$ variedades irredutíveis, com $V_{i}$ não contida em $V_{j}$, para todo $i \neq j$, tais que $V=V_{1} \cup \ldots \cup V_{p}$. Essas variedades são unicamente determinadas, a menos da ordem, e são chamadas de componentes irredutíveis de $V$.

Definição 1.9.7. Dizemos que um germe de espaço analítico $V$ é equidimensional quando todas as suas componentes irredutíveis tem a mesma dimensão.

Chamamos de germe de espaço analítico em $x$, um germe de conjunto $V$ em $x$ tal que, para alguma vizinhança $U$ de $x$, o germe $V \cap U$ pode ser descrito por $\mathcal{V}\left(f_{1}, \ldots, f_{r}\right)$, para alguns $f_{1}, \ldots, f_{r} \in \mathcal{O}_{n}$.

Definição 1.9.8. Dizemos que um germe $V=\mathcal{V}\left(f_{1}, \ldots, f_{r}\right)$ é reduzido se a $\mathbb{C}$-álgebra $\frac{\mathcal{O}_{n}}{\left\langle f_{1}, \ldots, f_{r}\right\rangle}$ não possui elementos nilpotentes.

Dizemos que um ponto $z$ de um germe de espaço analítico $V$ é um ponto regular ou suave se para alguma vizinhança $U$ de $z$, o germe $U \cap V$ pode ser descrito como o conjunto 
dos zeros de um número finito de germes de funções analíticas que possuem $z$ como ponto regular. Um ponto de $V$ não regular é chamado de ponto singular de $V$.

Teorema 1.9.3 (Hilbert's Nullstellensatz - versão local,[4]). Seja $I$ um ideal de $\mathcal{O}_{n}$. Então $\mathcal{I}(\mathcal{V}(I))=\operatorname{Rad}(I)$, onde $\operatorname{Rad}(I)$ é o ideal radical de $I$, ou seja, o ideal $\left\{f \in \mathcal{O}_{n}: \exists n \in\right.$ $\mathbb{N}$ tal que $\left.f^{n} \in I\right\}$.

\subsection{Estratificação de Whitney}

Introduziremos aqui a noção de estratificação de Whitney, introduzida por Whitney [28] e amplamente utilizada desde então. Uma referência para este assunto é [1].

Definição 1.10.1. Sejam $M$ uma variedade suave e $V \subset M$. Uma estratificação localmente finita de $V$ é uma partição de $V$ em subvariedades de $M$ (chamadas de estratos) tais que, para todo ponto de $V$ existe uma vizinhança em $M$ que encontra apenas um número finito de estratos.

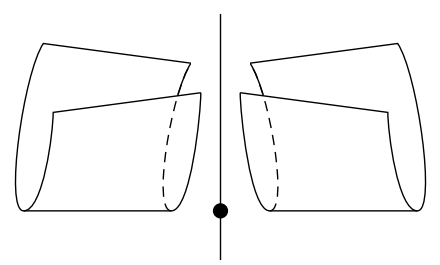

Definição 1.10.2. Dizemos que a estratificação $\left\{V_{\alpha}\right\}$ de $V$ satisfaz à condição de fronteira, se para dois estratos $V_{\alpha}$ e $V_{\beta}$, tais que $V_{\alpha} \cap \bar{V}_{\beta} \neq \emptyset$ então $V_{\alpha} \subset \bar{V}_{\beta}$.

\section{Condições de Whitney}

Definição 1.10.3. A estratificação $\left\{V_{\alpha}\right\}$ satisfaz às condições de Whitney se para todo $\operatorname{par}\left(V_{\alpha}, V_{\beta}\right)$ de estratos, tais que $V_{\beta}$ esteja no fecho de $V_{\alpha}$ e para todo ponto y de $V_{\beta}$ temos:

a) Para toda seqüência de pontos $x_{i}$ de $V_{\alpha}$ convergindo para $y$, tal que o limite

$$
\lim _{i \rightarrow \infty} T_{x_{i}}\left(V_{\alpha}\right)=T
$$


existe na Grassmanniana correspondente, então $T$ contém $T_{y}\left(V_{\beta}\right)$.

b) Se além disso temos uma seqüência $y_{i}$ de pontos de $V_{\beta}$ com limite y e tal que o limite de direções

$$
\lim _{i \rightarrow \infty} \overline{x_{i} y_{i}}=\lambda
$$

existe no espaço projetivo, então $T$ contém $\lambda$.

Estas são as chamadas condição (a) e condição (b) de Whitney.

Whitney mostrou em seu trabalho [28] que toda variedade analítica complexa admite uma estratificação satisfazendo essas duas condições.

Uma estratificação que satisfaz às condições de Whitney e à condição de fronteira é chamada de estratificação de Whitney, ou estratificação Whitney regular.

Exemplo 1.10.1. Consideremos como primeiro exemplo o cone $C$ com vértice na origem, e a estratificação $\left\{V_{1}, V_{2}\right\}$, onde $V_{1}$ é uma geratriz do cone e $V_{2}=C \backslash V_{1}$. Neste caso, as condições (a) e (b) de Whitney não são satisfeitas. Para ver isto, basta considerar uma seqüência $\left\{x_{i}\right\}$ de pontos de $C$, situados todos sobre uma geratriz $L$ do cone que não seja $V_{1}$, cujo limite seja a origem, de tal modo que o segmento $\overline{x_{i} y_{i}}$ tenha sempre a mesma direção $\lambda$.

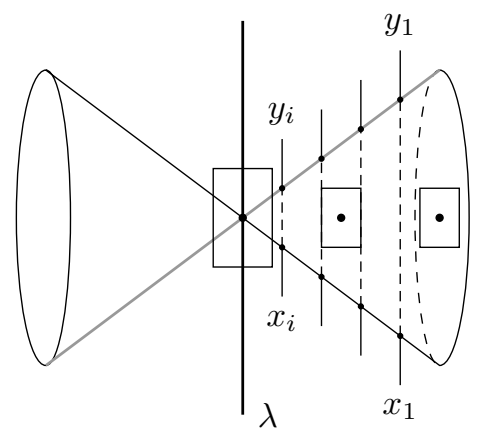

A condição (a) não é satisfeita, já que o limite dos espaços tangentes $T_{x_{i}} L$ não contém o espaço $T_{0}\left(V_{1}\right)$, a verificação de que a condição (b) também não é satisfeita vem do fato que $\lambda$ não está contida no limite dos espaços tangentes $T_{x_{i}} L$. 


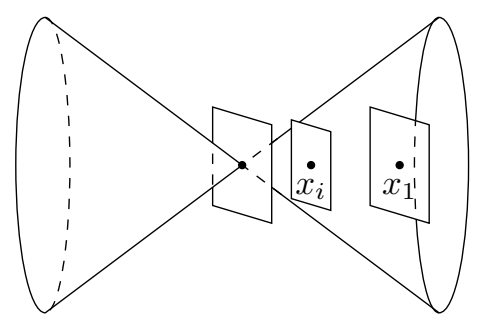

Exemplo 1.10.2. Consideremos agora a variedade $V$ em $\mathbb{C}^{3}$ dada pelo conjunto de zeros de $y^{2}+x^{3}-t^{2} x^{2}=0$.

Se tomarmos o eixo da coordenada t como um estrato $V_{1}$ e a parte regular da variedade $V_{\text {reg }}$ como sendo outro estrato, temos que a estratificação $\left\{V_{1}, V_{2}\right\}$ satisfaz a condição $(a)$, mas não a condição (b). Porém se acrescentarmos um estrato de dimensão zero, a origem de $\mathbb{C}^{3}$, teremos as duas condições de Whitney satisfeitas.

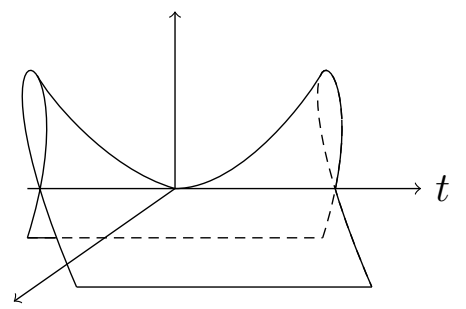

Figura 1.2: Variedade Singular.

\subsection{Teoria de Morse}

Apresentamos aqui os principais conceitos da teoria de Morse utilizada neste trabalho. Como referência sugerimos [18].

Seja M uma variedade diferenciável e $f: M \longrightarrow \mathbb{R}$ uma aplicação diferenciável $C^{\infty}$.

Definição 1.11.1. Um ponto $p \in M$ será um ponto crítico de $f$ se a derivada de $f$ no ponto $p$ for nula. Um ponto crítico será chamado não degenerado, se a matriz hessiana de $f$ em $p$ for não singular, isto é, se esta matriz tiver determinante não nulo. Os pontos críticos não degenerados são ditos pontos de Morse de $f$. 
Definição 1.11.2. A matriz hessiana define uma aplicação bilinear $H$ no espaço tangente $T_{p} M$. O índice desta aplicação é definido como sendo a dimensão do maior subespaço $V$ de $T_{p} M$ no qual $H$ é definida negativa e é chamado indice de Morse de $f$ em $p$.

Definição 1.11.3. Dizemos que uma aplicação $f$ é uma função de Morse se todas os seus pontos críticos são não degenerados, isto é se todos os pontos críticos forem pontos de Morse.

Lema 1.11.1 (Lema de Morse). Seja p um ponto crítico não degenerado de f. Então existe um sistema de coordenadas locais $\left(x_{1}, \ldots, x_{m}\right)$ em uma vizinhança $U$ de $p$ em $M$ tal que neste sistema de coordenadas $p=(0, \ldots, 0)$ e

$$
f(x)=f(p)-x_{1}^{2}-x_{2}^{2}-\ldots-x_{\lambda}^{2}+x_{\lambda+1}^{2}+\ldots x_{m}^{2},
$$

para todo $x=\left(x_{1}, \ldots, x_{m}\right) \in U$, em que $\lambda$ é o indice de Morse de $f$ em $p$. 



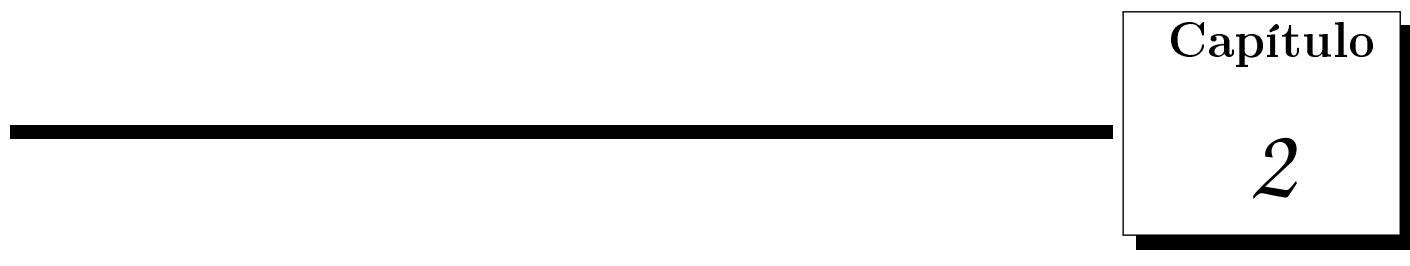

\section{O Teorema da Fibração}

As principais referências para este Capítulo são [19, 22].

Definição 2.0.4. O gradiente de uma função analítica $f: \mathbb{C}^{n+1} \rightarrow \mathbb{C}$ em um ponto $z \in \mathbb{C}^{n+1}$ é $a(n+1)$-upla

$$
\operatorname{grad} f(z)=\left(\overline{\frac{\partial f}{\partial z_{1}}}(z), \quad \ldots, \overline{\frac{\partial f}{\partial z_{n+1}}}(z)\right)
$$

em que a j-ésima componente é o complexo conjungado de $\frac{\partial f}{\partial z_{j}}(z)$.

Seja $p: I \rightarrow \mathbb{C}^{n+1}$ um caminho diferenciável. A derivada direcional de $f$ no ponto $p(t)$, na direção do vetor $\frac{d p}{d t}(t)$ é dada por:

$$
\frac{d}{d t}(f \circ p)(t)=d f(p(t)) \cdot \frac{d p}{d t}(t)
$$

Em termos dos elementos da matriz $\left(\frac{\partial f}{\partial z_{j}}(z)\right)_{1 \times(n+1)}$ de $d f(z)$ e dos elementos da matriz $\left(v_{1} \ldots v_{n+1}\right)$ de $\frac{d p}{d t}(t)$, podemos escrever:

$$
\frac{d}{d t}(f \circ p)(t)=\sum_{j=1}^{n+1} \frac{\partial f}{\partial z_{j}}(p(t)) \cdot v_{j}=\left\langle\frac{d p}{d t}(t), \operatorname{grad} f(p(t))\right\rangle,
$$

em que $\langle$,$\rangle é o produto interno hermitiano$ 


$$
\begin{aligned}
\langle,\rangle: \mathbb{C}^{n+1} \times \mathbb{C}^{n+1} & \longrightarrow \mathbb{C} \\
(a, b) & \longmapsto \sum_{j=1}^{n+1} a_{j} \overline{b_{j}} .
\end{aligned}
$$

Assim, definição de $\operatorname{grad} f(z)$ é escolhida de maneira que a regra da cadeia para a derivada de $f$ ao longo de um caminho $z=p(t)$ é da forma

$$
\frac{d}{d t}(f \circ p)(t)=\left\langle\frac{d p}{d t}(t), \operatorname{grad} f(p(t))\right\rangle
$$

Em outras palavras, a derivada direcional de $f$ na direção de um vetor $v$, em um ponto $z$, pode ser expressa pelo produto interno

$$
d f(z) \cdot v=\langle v, \operatorname{grad} f(z)\rangle .
$$

Agora, seja $K$ a interseção dos zeros de $f$ com a esfera $S_{\varepsilon}=\left\{z \in \mathbb{C}^{n+1} ;\|z\|=\varepsilon\right\}$. Considere a aplicação $\phi: S_{\varepsilon} \backslash K \longrightarrow S^{1}$ definida por

$$
\phi(z)=\frac{f(z)}{|f(z)|}
$$

Note que $S_{\varepsilon} \backslash K$ é uma variedade diferenciável real de dimensão $2 n+1$.

O próximo lema fornece uma caracterização dos pontos críticos de $\phi$.

Lema 2.0.2. Os pontos críticos de $\phi$ são os pontos $z \in S_{\varepsilon} \backslash K$ para os quais o vetor $i \operatorname{grad} \log f(z)$ é um múltiplo real de z.

Antes de demonstrarmos o Lema 2.0.2. faremos as seguintes observações:

Observação 2.0.3. Para $z \in S_{\varepsilon} \backslash K$, isto é $f(z) \neq 0$, podemos escrever $\phi(z)=\frac{f(z)}{|f(z)|}=$ $e^{i \theta(z)}$ e escolher, localmente, uma determinação para $\log f(z)$ e $\theta(z)$. Nesta caso,

$$
i \theta(z)=\log \left(\frac{f(z)}{|f(z)|}\right)=\log f(z)-\log |f(z)|
$$

o que implica que

$$
\theta(z)=\boldsymbol{R} e(-i \log f(z))
$$


Ao longo deste trabalho vamos considerar sempre uma determinação local para o logarítmo e para a função $\theta$.

Observação 2.0.4. Embora a função $\log f(z)$ possa ser definida apenas localmente, seu gradiente pode ser estendido naturalmente para todo $z \in \mathbb{C}^{n+1} \backslash f^{-1}(0)$. Basta notar que

$\operatorname{grad} \log f(z)=\left(\overline{\frac{\partial}{\partial z_{1}} \log f(z)}, \ldots, \overline{\frac{\partial}{\partial z_{n+1}} \log f(z)}\right)=\left(\frac{1}{\overline{f(z)}} \frac{\overline{\partial f}}{\partial z_{1}}(z), \ldots, \frac{1}{\overline{f(z)}} \overline{\frac{\partial f}{\partial z_{n+1}}}(z)\right)$

Ou seja,

$$
\operatorname{grad} \log f(z)=\frac{\operatorname{grad} f(z)}{\overline{f(z)}} .
$$

Observação 2.0.5. O espaço vetorial hermitiano $\mathbb{C}^{n+1}$ pode ser pensado como um espaço vetorial euclidiano de dimensão $2 n+2$, basta tomar o produto interno de $z, z^{\prime} \in \mathbb{C}^{n+1}$ como sendo $\boldsymbol{R e}\left\langle z, z^{\prime}\right\rangle$.

De fato, consideremos o isomorfismo $\gamma: \mathbb{C}^{n+1} \longrightarrow \mathbb{R}^{2 n+2}$ definido por

$$
\gamma\left(a_{1}+i b_{1}, \ldots, a_{n+1}+i b_{n+1}\right)=\left(a_{1}, b_{1}, \ldots, a_{n+1}, b_{n+1}\right)
$$

Sejam $z=\left(a_{1}+i b_{1}, \ldots, a_{n+1}+i b_{n+1}\right)$ e $z^{\prime}=\left(a_{1}^{\prime}+i b_{1}^{\prime}, \ldots, a_{n+1}^{\prime}+i b_{n+1}^{\prime}\right)$. Então:

$$
\boldsymbol{R} \boldsymbol{e}\left\langle z, z^{\prime}\right\rangle=\boldsymbol{R} \boldsymbol{e}\left(\sum_{j=1}^{n+1}\left(a_{j}+i b_{j}\right) \cdot\left(a_{j}^{\prime}-i b_{j}^{\prime}\right)\right)=\sum_{j=1}^{n+1}\left(a_{j} a_{j}^{\prime}+b_{j} b_{j}^{\prime}\right)=\left(\gamma(z), \gamma\left(z^{\prime}\right)\right)
$$

em que ( , ) denota o produto interno usual em $\mathbb{R}^{2 n+2}$.

Podemos demonstrar agora o Lema 2.0.2.

Demonstração do Lema 2.0.2 Como $\operatorname{dim} T_{\phi(z)} S^{1}=1$, a derivada

$$
d \phi(z): T_{z}\left(S_{\varepsilon} \backslash K\right) \longrightarrow T_{\phi(z)} S^{1}
$$

não será sobrejetora, isto é, $z$ será ponto crítico de $\phi$, se, e somente se, $d \phi(z)$ for a aplicação nula.

Escrevendo $\phi(z)=e^{i \theta(z)}$, sabemos, pela Observação 2.0.3. que $\theta(z)=\mathbf{R e}(-i \log f(z))$. Derivando-se a função $\theta$ ao longo de uma curva $z=p(t)$, obtemos: 


$$
\begin{aligned}
\frac{d}{d t}(\theta \circ p)(t) & =\boldsymbol{R e}\left(-i \frac{d}{d t}(\log f(p(t)))\right. \\
& =\boldsymbol{R e}\left(\frac{-i}{f(p(t))} \frac{d}{d t}(f \circ p)(t)\right) \\
& =\boldsymbol{R e}\left(\frac{-i}{f(p(t))}\left\langle\frac{d p}{d t}(t), \operatorname{grad} f(p(t))\right\rangle\right) \\
& =\boldsymbol{R e}\left\langle\frac{d p}{d t}(t), i \operatorname{grad} \log f(p(t))\right\rangle .
\end{aligned}
$$

Em outras palavras, a derivada direcional de $\theta$, no ponto $z=p(t)$, na direção do vetor $v=\frac{d p}{d t}(t)$, é dada por

$$
d \theta(z) \cdot v=\mathbf{R e}\langle v, i \operatorname{grad} \log f(z)\rangle .
$$

Agora, se o vetor $i \operatorname{grad} \log f(z)$ for um múltiplo real de $z$ (isto é, se este vetor for normal a $S_{\varepsilon}$ ), pela Observação 2.0.5 para todo vetor $v$ tangente a $S_{\varepsilon} \backslash K \mathrm{em} z$, a derivada direcional $\operatorname{Re}\langle v, i \operatorname{grad} \log f(z)\rangle$ será nula. Como

$$
\phi(z)=e^{i \theta(z)} \Rightarrow d \phi(z)=\phi(z) d \theta(z)
$$

segue que, para todo $v \in T_{z}\left(S_{\varepsilon} \backslash K\right)$,

$$
d \phi(z) \cdot v=\phi(z) d \theta(z) \cdot v=0
$$

Por outro lado, se os vetores $z$ e $i \operatorname{grad} \log f(z)$ forem linearmente independentes sobre os reais, então existirá um vetor $v$ em nosso espaço euclidiano de maneira que

$$
\left\{\begin{array}{l}
\boldsymbol{\operatorname { R e }}\langle v, z\rangle=0 \\
\boldsymbol{\operatorname { R e }}\langle v, i \operatorname{grad} \log f(z)\rangle \neq 0
\end{array}\right.
$$

De fato, se $\boldsymbol{R e}\langle i \operatorname{grad} \log f(z), z\rangle=0$, basta escolher

$$
v=i \operatorname{grad} \log f(z),
$$

que é não nulo, pois estamos considerando $i \operatorname{grad} \log f(z)$ e $z$ linearmente independentes sobre $\mathbb{R}$. 
Se, por outro lado, $\operatorname{Re}\langle i \operatorname{grad} \log f(z), z\rangle \neq 0$, tomamos

$$
v=\mathrm{i} \operatorname{grad} \log f(z)-\frac{\operatorname{Re}\langle i \operatorname{grad} \log f(z), z\rangle z}{\operatorname{Re}\langle z, z\rangle} .
$$

Neste caso, é fácil ver que $\boldsymbol{R e}\langle v, z\rangle=0$. Também, calculando $\boldsymbol{R e}\langle v, v\rangle$, obtemos $\boldsymbol{\operatorname { R e }}\langle v, v\rangle=\boldsymbol{\operatorname { R e }}\langle v, i \operatorname{grad} \log f(z)\rangle . \quad$ Portanto, $\boldsymbol{\operatorname { R e }}\langle v, i \operatorname{grad} \log f(z)\rangle \neq 0$, já que o vetor $v$ escolhido é não nulo.

Agora, pela Observação 2.0.5. $\operatorname{Re}\langle v, z\rangle=0$ implica que $v \in T_{z}\left(S_{\varepsilon} \backslash K\right)$. Além disso, $d \theta(z) \cdot v=\operatorname{Re}\langle v, i \operatorname{grad} \log f(z)\rangle \neq 0$, isto é, $d \phi(z) \cdot v=\phi(z) d \theta(z) \cdot v \neq 0$. Portanto, $z$ não é ponto crítico de $\phi$. Isto conclui a demonstração do lema.

A partir deste ponto, vamos supor que $f: \mathbb{C}^{n+1} \rightarrow \mathbb{C}$ é uma função polinomial não nula que se anula na origem de $\mathbb{C}^{n+1}$. Queremos provar que, para $\varepsilon>0$ suficientemente pequeno, a aplicação $\phi: S_{\varepsilon} \backslash K \rightarrow S^{1}$ não admite pontos críticos.

Vamos denotar por $V$ a hipersuperfície $f^{-1}(0) \subset \mathbb{C}^{n+1}$.

Lema 2.0.6. Existe $\varepsilon>0$ tal que, para todo $z \in \mathbb{C}^{n+1}-V$ com $\|z\| \leq \varepsilon$ e z no dominio de i grad $\log f$, os vetores z e $i \operatorname{grad} \log f(z)$ são linearmente independentes sobre os reais.

Vamos demonstrar um resultado um pouco mais forte:

Lema 2.0.7. Seja $f: \mathbb{C}^{n+1} \rightarrow \mathbb{C}$ uma função polinomial não nula que se anula na origem. Existe $\varepsilon_{0}>0$ tal que, para todo $z \in \mathbb{C}^{n+1}-V$ com $\|z\| \leq \varepsilon_{0}$ e z no dominio de i grad $\log f$, os vetores z e $\operatorname{grad} \log f(z)$ são linearmente independentes sobre os complexos ou

$$
\operatorname{grad} \log f(z)=\lambda z
$$

em que $\lambda$ é um número complexo não nulo, cujo argumento $(\arg \lambda)$ em valor absoluto é menor que, digamos, $\pi / 4$. Em outras palavras, $\lambda$ está no quadrante aberto de $\mathbb{C}$ simétrico em relação ao semi-eixo real positivo.

Observe que, desta maneira, a prova do Lema 2.0.6 segue diretamente do Lema 2.0.7: Primeiramente, se $z$ e grad $\log f(z)$ forem linearmente independentes sobre os complexos, também o serão $z$ e i grad $\log f(z)$. Porém, se grad $\log f(z)=\lambda z \operatorname{com} \lambda \in \mathbb{C} \backslash\{0\}$ 
e $|\arg \lambda|<\pi / 4$, então $\lambda$ não será imaginário puro e, portanto, i grad $\log f(z)=(i \lambda) z$ $\operatorname{com} i \lambda \in \mathbb{C} \backslash \mathbb{R}$.

A demonstração do Lema 2.0.7 dependerá do Lema de Seleção da Curva e do Lema 2.0 .9 apresentados a seguir.

Lema 2.0.8 (Lema de Seleção da Curva [19]). Seja $V \subset \mathbb{R}^{m}$ um conjunto algébrico real, e seja $U \subset \mathbb{R}^{m}$ um conjunto aberto definido por um número finito de desigualdades:

$$
U=\left\{x \in \mathbb{R}^{n+1} ; g_{1}(x)>0, \ldots, g_{k}(x)>0\right\}
$$

isto é, um conjunto semi algébrico. Se $U \cap V$ contém pontos arbitrariamente próximos da origem, isto é, se $0^{\prime}$ pertence ao fecho de $U \cap V$, então existe uma curva analítica real

$$
p:[0, \delta) \longrightarrow \mathbb{R}^{m}
$$

satisfazendo $p(0)=0^{\prime}$ e $p(t) \in U \cap V$ para todo $t>0$.

Lema 2.0.9. Seja $p:\left[0, \delta^{\prime}\right) \longrightarrow \mathbb{C}^{n+1}$ um caminho analítico real com $p(0)=0$, tal que, para cada $t>0$, o número $f(p(t))$ é não nulo e o vetor grad $\log f(p(t))$ é um múltiplo complexo de $p(t)$, isto é,

$$
\operatorname{grad} \log f(p(t))=\lambda(t) p(t), \quad \lambda(t) \in \mathbb{C}
$$

Então $\arg \lambda(t) \rightarrow 0$ quando $t \rightarrow 0$.

Em outras palavras, $\lambda(t)$ é não nulo para valores positivos de $t$ suficientemente pequenos e

$$
\lim _{t \rightarrow 0} \frac{\lambda(t)}{|\lambda(t)|}=\lim _{t \rightarrow 0} e^{i \arg \lambda(t)}=1
$$

Demonstração. Como $p(t), f(p(t))$ e grad $f(p(t))$ são funções analíticas reais, podemos considerar suas expansões em séries de Taylor em torno da origem:

$$
\begin{array}{clc}
p(t) & =a_{0} t^{\alpha}+a_{1} t^{\alpha+1}+a_{2} t^{\alpha+2}+\ldots, \quad a_{i} \in \mathbb{C}^{n+1} ; \\
f(p(t)) & =b_{0} t^{\beta}+b_{1} t^{\beta+1}+b_{2} t^{\beta+2}+\ldots, \quad b_{i} \in \mathbb{C} ; \\
\operatorname{grad} f(p(t)) & =c_{0} t^{\gamma}+c_{1} t^{\gamma+1}+c_{2} t^{\gamma+2}+\ldots, \quad c_{i} \in \mathbb{C}^{n+1}
\end{array}
$$


em que $a_{0}, b_{0}$ e $c_{0}$ são não nulos.

A identidade $\frac{d}{d t}(f \circ p)(t)=\left\langle\frac{d p}{d t}(t), \operatorname{grad} f(p(t))\right\rangle$ nos mostra que grad $f(p(t))$ não pode ser identicamente nulo, caso contrário, teríamos $\frac{d}{d t}(f \circ p)(t)=0$, o que implicaria $f \circ p$ constante em uma vizinhança de $t=0$. Como $f(p(0))=f(0)=0, f(p(t))$ seria nula nesta vizinhança, contrariando a hipótese do lema.

Observe também que os expoentes $\alpha, \beta$ e $\gamma$ são inteiros positivos com $\alpha \geq 1$ (pois $p(0)=0$ ) e $\beta \geq 1$ (pois $f(0)=0$ ). Além disso, as séries são todas convergentes para, digamos, $|t|<\varepsilon^{\prime}$.

Por hipótese, para cada $t>0$, temos grad $\log f(p(t))=\lambda(t) p(t)$, então

$$
\operatorname{grad} f(p(t))=\lambda(t) p(t) \overline{f(p(t))}
$$

Como as componentes dos vetores grad $f(p(t))$ e $p(t) \overline{f(p(t))}$ são funções analíticas reais, comparando as componentes correspondentes, podemos escrever $\lambda(t)$ como um quociente de funções analíticas reais, admitindo portanto, expansão em série de Laurent. Considerando-se as expansões de Taylor na igualdade $\operatorname{grad} f(p(t))=\lambda(t) p(t) \overline{f(p(t))}$, obtemos:

$$
c_{0} t^{\gamma}+\ldots=\lambda(t)\left(a_{0} \overline{b_{0}} t^{\alpha+\beta}+\ldots\right)
$$

Assim, o desenvolvimento em série de Laurent de $\lambda$ é da forma:

$$
\lambda(t)=\lambda_{0} t^{\gamma-\alpha-\beta}\left(1+k_{1} t+k_{2} t^{2}+\ldots\right)
$$

com $c_{0}=\lambda_{0} a_{0} \overline{b_{0}}$. Substituindo-se a equação na expansão em série de potências da identidade

$$
\frac{d}{d t}(f \circ p)(t)=\left\langle\frac{d p}{d t}(t), \operatorname{grad} f(p(t))\right\rangle,
$$

temos

$$
\beta b_{0} t^{\beta-1}+\ldots=\left\langle\alpha a_{0} t^{\alpha-1}+\ldots, \lambda_{0} a_{0} \overline{b_{0}} t^{\gamma}+\ldots\right\rangle=\alpha\left\|a_{0}\right\|^{2} \overline{\lambda_{0}} b_{0} t^{\alpha-1+\gamma}+\ldots
$$


Comparando-se os coeficientes principais, segue que $\beta=\alpha\left\|a_{0}\right\|^{2} \overline{\lambda_{0}}$. Isto é, $\lambda_{0}$ é um número real positivo.

Para calcularmos $\lim (\lambda(t) /|\lambda(t)|)$ é necessário assegurar que $\lambda(t) \neq 0$ para $t \neq 0 \mathrm{em}$ alguma vizinhança de zero. Esta condição será satisfeita devido à igualdade

$$
\lambda(t)=\lambda_{0} t^{\gamma-\alpha-\beta}\left(1+k_{1} t+k_{2} t^{2}+\ldots\right) .
$$

De fato, como $\left(1+k_{1} t+k_{2} t^{2}+\ldots\right)$ é contínua e tem limite 1 quando $t \rightarrow 0$, dado $\varepsilon^{\prime \prime}>0$ suficientemente pequeno, existe uma vizinhança de $t=0$ na qual $\left(1+k_{1} t+\ldots\right)>\varepsilon^{\prime \prime}$. Logo,

$$
\lambda(t)=\lambda_{0} t^{\gamma-\alpha-\beta}\left(1+k_{1} t+k_{2} t^{2}+\ldots\right)>\varepsilon^{\prime \prime} \lambda_{0} t^{\gamma-\alpha-\beta}
$$

para $t \neq 0$ nesta vizinhança. Mas, como $\lambda_{0}>0$, concluímos que

$$
\lim _{t \rightarrow 0} \frac{\lambda(t)}{|\lambda(t)|}=\lim _{t \rightarrow 0} \frac{\lambda_{0} t^{\gamma-\alpha-\beta}\left(1+k_{1} t+k_{2} t^{2}+\ldots\right)}{\left|\lambda_{0} t^{\gamma-\alpha-\beta}\right|\left|\left(1+k_{1} t+k_{2} t^{2}+\ldots\right)\right|}=1
$$

Demonstração do Lema 2.0.7 Suponha primeiramente que existam pontos $z \in \mathbb{C}^{n+1} \backslash V$, arbitrariamente próximos da origem, e escalares $\lambda \in \mathbb{C}$, com $\operatorname{grad} \log f(z)=\lambda z \neq 0$ e $|\arg \lambda|>\pi / 4$. Isto é, suponha que $\lambda$ pertença ao semi-plano aberto

$$
\boldsymbol{R e}((1+i) \lambda)<0
$$

ou ao semi-plano aberto

$$
\boldsymbol{\operatorname { R e }}((1-i) \lambda)<0
$$

Queremos expressar estas condições por meio de equações e inequações polinomiais, para aplicarmos então o Lema de Seleção da Curva.

Seja $W$ o conjunto de todos os $z \in \mathbb{C}^{n+1}$ para os quais os vetores $\operatorname{grad} f(z)$ e $z$ são linearmente dependentes sobre $\mathbb{C}$. Então, $z \in W$ se, e somente se,

$$
z_{j} \overline{\frac{\partial f}{\partial z_{k}}}=z_{k} \overline{\frac{\partial f}{\partial z_{j}}}, \quad j, k=1, \ldots, n+1
$$


De fato, se para algum $\lambda \in \mathbb{C}$ tivermos

$$
\left(\overline{\frac{\partial f}{\partial z_{1}}(z)}, \ldots, \overline{\frac{\partial f}{\partial z_{n+1}}(z)}\right)=\lambda\left(z_{1}, \ldots, z_{n+1}\right)
$$

segue que

$$
z_{k} \overline{\frac{\partial f}{\partial z_{j}}(z)}=\lambda z_{k} z_{j}=z_{j} \overline{\frac{\partial f}{\partial z_{k}}(z)}, \quad j, k=1, \ldots, n+1
$$

Por outro lado, se $z \neq 0$, então $z=\left(z_{1}, \ldots, z_{n+1}\right)$ com $z_{s} \neq 0$, para algum $s$. Assim, podemos escrever

$$
\frac{z_{j}}{z_{s}} \overline{\frac{\partial f}{\partial z_{s}}(z)}=\overline{\frac{\partial f}{\partial z_{j}}(z)}, \quad j=1, \ldots, n+1
$$

$\log \mathrm{o}$

$$
\left(\overline{\frac{\partial f}{\partial z_{1}}(z)}, \ldots, \overline{\frac{\partial f}{\partial z_{n+1}}(z)}\right)=\left(\frac{z_{1}}{z_{s}} \overline{\frac{\partial f}{\partial z_{s}}(z)}, \ldots, \frac{z_{n+1}}{z_{s}} \overline{\frac{\partial f}{\partial z_{s}}(z)}\right)=\frac{1}{z_{s}} \overline{\frac{\partial f}{\partial z_{s}}(z)}\left(z_{1}, \ldots, z_{n+1}\right)
$$

Ou seja, grad $f(z)$ e $z$ são linearmente dependentes sobre $\mathbb{C}$. (Note que, se $z=0 \in \mathbb{C}^{n+1}$, então $z$ pertence trivialmente a $W$.)

Escrevendo $z_{j}=x_{j}+i y_{j}$ e tomando as partes real e imaginária das equações

$$
z_{j} \overline{\frac{\partial f}{\partial z_{k}}(z)}=z_{k} \overline{\frac{\partial f}{\partial z_{j}}(z)},
$$

obtemos (lembrando que fé polinomial) uma coleção de equações polinomiais nas variáveis reais $x_{j}$ e $y_{j}$. Isto mostra que $W \subset \mathbb{C}^{n+1}$ é um conjunto algébrico real.

Notemos que um ponto $z \in \mathbb{C}^{n+1} \backslash V$ pertence a $W$ se, e somente se,

$$
\frac{\operatorname{grad} f(z)}{\overline{f(z)}}=\lambda z,
$$

para algum $\lambda \in \mathbb{C}$, isto é,

$$
\operatorname{grad} f(z)=\lambda \overline{f(z)} z
$$

Tomando o produto interno por $\overline{f(z)} z$, obtemos

$$
\langle\operatorname{grad} f(z), \overline{f(z)} z\rangle=\lambda\|\overline{f(z)} z\|^{2}
$$


Em outras palavras, o número $\lambda$ multiplicado por um número real positivo é igual a $\lambda^{\prime}(z)=\langle\operatorname{grad} f(z), \overline{f(z)} z\rangle$, e portanto,

$$
\arg \lambda=\arg \lambda^{\prime}
$$

Note que $\lambda^{\prime}$ é uma função polinomial, a valores complexos, nas variáveis reais $x_{j}$ e $y_{j}$.

Agora, denotemos por $U_{+}$(respectivamente $U_{-}$) o conjunto aberto constituído de todos os $z \in \mathbb{C}^{n+1}$ que satisfazem a desigualdade

$$
\boldsymbol{\operatorname { R e }}\left((1+i) \lambda^{\prime}(z)\right)<0
$$

(respectivamente $\left.\boldsymbol{R e}\left((1-i) \lambda^{\prime}(z)\right)<0\right)$.

Observe que se $z \in V$, isto é $f(z)=0$, então $\lambda^{\prime}(z)=0$ e $z$ não pertence a $U_{+}$nem a $U_{-}$. Ou seja, $U_{+} \subset \mathbb{C}^{n+1} \backslash V$ e $U_{-} \subset \mathbb{C}^{n+1} \backslash V$.

Escrevendo cada componente $z_{j}$ de $z$ como $z_{j}=x_{j}+i y_{j}$ em $\lambda^{\prime}(z)=\langle\operatorname{grad} f(z), \overline{f(z)} z\rangle$ e substituindo-as nas expressões para $U_{+}$e $U_{-}$, verifica-se que estes são conjuntos semialgébricos reais.

A hipótese de existirem $z \in \mathbb{C}^{n+1} \backslash V$ arbtrariamente próximos da origem, com $\operatorname{grad} \log f(z)=\lambda z \neq 0$ e $|\arg \lambda|>\pi / 4$ é então equivalente a

$$
z \in W \cap\left(U_{+} \cup U_{-}\right)=\left(W \cap U_{+}\right) \cup\left(W \cap U_{-}\right)
$$

e

$$
0 \in \overline{W \cap\left(U_{+} \cup U_{-}\right)}=\overline{W \cap U_{+}} \cup \overline{W \cap U_{-}},
$$

em que barra denota o fecho do conjunto.

Portanto, pelo Lema de Seleção da Curva, deve existir um caminho analítico real

$$
p:[0, \delta) \longrightarrow \mathbb{C}^{n+1}
$$


$\operatorname{com} p(0)=0$ e com $p(t) \in W \cap U_{+}$, ou $p(t) \in W \cap U_{-}$, para todo $t>0$. Em qualquer dos casos, obtemos

$\operatorname{grad} \log f(p(t))=\lambda(t) p(t), \operatorname{com}|\arg \lambda(t)|>\pi / 4$, para todo $t>0$

O que não pode ocorrer, pois $p(t) \in\left(U_{+} \cup U_{-}\right)$implica $p(t) \in \mathbb{C}^{n+1} \backslash V$, para $t$ não nulo, de onde segue que $f(p(t)) \neq 0$, para todo $t \neq 0$. Então poderíamos aplicar o Lema 2.0.9 para obtermos uma contradição.

Esta contradição não completa a demonstração: permanece a possibilidade de existirem pontos $z \in \mathbb{C}^{n+1}-V$, arbitrariamente próximos da origem, satisfazendo

$$
\operatorname{grad} \log f(z)=\lambda z, \operatorname{com} \lambda=0 \text { ou }|\arg \lambda|=\pi / 4
$$

Neste caso, definimos o conjuno algébrico real

$$
\tilde{W}=\left\{z \in \mathbb{C}^{n+1} ;\left(\boldsymbol{R e}\left((1+i) \lambda^{\prime}(z)\right)\right) \cdot(\boldsymbol{R e}((1-i) \lambda(z)))=0\right\}
$$

e o conjunto semi-algébrico real

$$
U=\left\{z \in \mathbb{C}^{n+1} ;\|f(z)\|^{2}>0\right\}
$$

Estamos supondo que $z \in(W \cap \tilde{W}) \cap U$ e $0 \in \overline{(W \cap \tilde{W}) \cap U}$. Novamente, pelo Lema de Seleção da Curva, deve existir um caminho real analítico $p:[0, \delta) \longrightarrow \mathbb{C}^{n+1}$ tal que $p(0)=0$ e $p(t) \in(W \cap \tilde{W}) \cap U$, para todo $t>0$.

Agora, $p(t) \in W$ implica que $\operatorname{grad} \log f(p(t))=\lambda(t) p(t)$ e $p(t) \in \tilde{W}$ implica que $\lambda(t)=\lambda^{\prime}(t)=0$ ou $|\arg \lambda(t)|=\left|\arg \lambda^{\prime}(t)\right|=\pi / 4, t>0$. Como também temos $p(t) \in U$, então $f(p(t)) \neq 0$, para $t>0$, e podemos aplicar o Lema 2.0.9 para novamente obtermos uma contradição.

Agora, usando o Lema 2.0 .2 obtemos o seguinte corolário:

Corolário 2.0.10. Nas condiçôes do Lema 2.0.7 se $\varepsilon \leq \varepsilon_{0}$, a aplicação $\phi: S_{\varepsilon} \backslash K \longrightarrow S^{1}$ não terá pontos críticos. 
Assim, para cada $e^{i \theta} \in S^{1}$, o espaço

$$
F_{\theta}=\phi^{-1}\left(e^{i \theta}\right) \subset S_{\varepsilon}-K
$$

é uma variedade diferenciável de dimensão real $2 n$.

Definição 2.0.5. O número $\varepsilon_{0}$ acima é chamado raio de Milnor de $\phi$. A variedade $F_{\theta}$, é dita fibra de Milnor da aplicação $\phi$.

Para demonstrarmos que $\phi$ é de fato a projeção de uma fibração localmente trivial, faremos um uso mais refinado do Lema 2.0.7. de modo a controlar cuidadosamente o comportamento de $\phi(z)$ quando $z$ tende ao conjunto $K$, onde $\phi$ não está definida.

Lema 2.0.11. Se $\varepsilon \leq \varepsilon_{0}$, então existe um campo de vetores diferenciável, $v(z)$, tangente $a S_{\varepsilon} \backslash K$ tal que, para cada z em $S_{\varepsilon} \backslash K$, o produto interno hermitiano

$$
\langle v(z), i \operatorname{grad} \log f(z)\rangle
$$

é não nulo e tem argumento em valor absoluto menor que $\pi / 4$.

Demonstração. Iniciaremos a demonstração construindo campos de vetores em $S_{\varepsilon} \backslash K$ localmente: dado um ponto $z_{a}$ em $S_{\varepsilon} \backslash K$, exibiremos um campo $v_{a}(z)$ ao longo de uma vizinhança $U_{a}$ de $z_{a}$ de maneira que as propriedades do teorema sejam satisfeitas. Em seguida, usaremos uma partição da unidade para obtermos $v(z)$.

Seja $z_{a} \in S_{\varepsilon} \backslash K$, consideremos os casos:

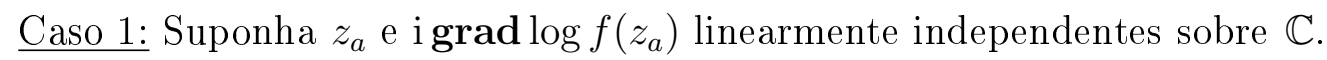

Então, as equações

$$
\begin{aligned}
& \left\langle v, z_{a}\right\rangle=0, \\
& \left\langle v, \text { i grad } \log f\left(z_{a}\right)\right\rangle=1
\end{aligned}
$$

admitem soluções simultâneas $v$, pois, substituindo $v=\left(v_{1}, \ldots, v_{n+1}\right), z_{a}=\left(z_{1}^{a}, \ldots, z_{n+1}^{a}\right)$ e i grad $\log f\left(z_{a}\right)=\left(u_{1}, \ldots, u_{n+1}\right)$ nas identidades, obtemos um sistema de duas equações lineares com $n+1$ variáveis. 
Em particular, existe uma única solução simultânea no espaço gerado pelos vetores $z_{a}$ e i grad $\log f\left(z_{a}\right)$. Isto é, uma única solução da forma

$$
v=\alpha z_{a}+\beta \text { i grad } \log f\left(z_{a}\right), \quad \operatorname{com} \alpha, \beta \in \mathbb{C}
$$

De fato, como $z_{a}$ e i grad $\log f\left(z_{a}\right)$ são linearmente independentes sobre $\mathbb{C}$, o determinante de Gram [23],

$$
\operatorname{det} G=\left|\begin{array}{cc}
\left\langle z_{a}, z_{a}\right\rangle & \left\langle i \operatorname{grad} \log f\left(z_{a}\right), z_{a}\right\rangle \\
\left\langle z_{a}, \mathrm{i} \operatorname{grad} \log f\left(z_{a}\right)\right\rangle & \left\langle\mathrm{i} \operatorname{grad} \log f\left(z_{a}\right), \mathrm{i} \operatorname{grad} \log f\left(z_{a}\right)\right\rangle
\end{array}\right|
$$

é não nulo. Logo, o sistema linear, nas variáveis $\alpha$ e $\beta$,

$$
\left\{\begin{array}{l}
\left\langle v, z_{a}\right\rangle=\alpha\left\langle z_{a}, z_{a}\right\rangle+\beta\left\langle i \operatorname{grad} \log f\left(z_{a}\right), z_{a}\right\rangle=0 \\
\left\langle v, \text { i grad } \log f\left(z_{a}\right)\right\rangle=\alpha\left\langle z_{a}, \text { i grad } \log f\left(z_{a}\right)\right\rangle+\beta\left\langle i \operatorname{grad} \log f\left(z_{a}\right), \text { i grad } \log f\left(z_{a}\right)\right\rangle=1
\end{array}\right.
$$

tem uma única solução dada por

$$
\begin{aligned}
& \alpha=\frac{-1}{\operatorname{det} G}\left\langle\operatorname{igrad} \log f\left(z_{a}\right), z_{a}\right\rangle \\
& \beta=\frac{1}{\operatorname{det} G}\left\langle z_{a}, z_{a}\right\rangle
\end{aligned}
$$

portanto,

$$
v=\frac{1}{\operatorname{det} G}\left[\left\langle z_{a}, z_{a}\right\rangle \mathrm{i} \operatorname{grad} \log f\left(z_{a}\right)-\left\langle\operatorname{igrad} \log f\left(z_{a}\right), z_{a}\right\rangle z_{a}\right]
$$

Agora, observe que a função $h: S_{\varepsilon} \backslash K \longrightarrow \mathbb{R}$ definida por

$$
h(z)=\operatorname{det} G(z)=\|z\|^{2}\|\mathrm{i} \operatorname{grad} \log f(z)\|^{2}-|\langle z, \operatorname{igrad} \log f(z)\rangle|^{2}
$$

é uma função $C^{\infty}$. Então, como $h\left(z_{a}\right) \neq 0$, existe uma vizinhança $U_{a}^{1}$ de $z_{a}$ tal que $h(z) \neq 0$ para todo $z$ em $U_{a}^{1}$. Além disso, a função $g: S_{\varepsilon} \backslash K \longrightarrow \mathbb{C}$ definida por

$$
g(z)=\|z\|^{2} \mathrm{i} \operatorname{grad} \log f(z)-\langle i \operatorname{grad} \log f(z), z\rangle z
$$


é $C^{\infty}$ e não nula em $z_{a}$. Logo, existe uma vizinhança $U_{a}^{2}$ de $z_{a}$ tal que $g(z) \neq 0$, para todo $z$ em $U_{a}^{2}$. Assim, podemos definir

$$
v_{a}(z)=\frac{\|z\|^{2} \mathrm{i} \operatorname{grad} \log f(z)-\langle i \operatorname{grad} \log f(z), z\rangle z}{\|z\|^{2}|| \mathrm{i} \operatorname{grad} \log f(z) \|^{2}-|\langle z, \operatorname{igrad} \log f(z)\rangle|^{2}},
$$

para todo $z \in U_{a}=U_{a}^{1} \cap U_{a}^{2}$.

Por construção, para todo $z \in U_{a}$, obtemos

$$
\begin{aligned}
& \left\langle v_{a}(z), z\right\rangle=0, \\
& \left\langle v_{a}(z), \text { i grad } \log f(z)\right\rangle=1 .
\end{aligned}
$$

A primeira equação garante que $\boldsymbol{R e}\left\langle v_{a}(z), z\right\rangle=0$, isto é, que $v_{a}(z)$ é tangente a $S_{\varepsilon} \backslash K$ em $z$.

Caso 2: Se $\operatorname{grad} \log f\left(z_{a}\right)$ for igual a $\lambda z_{a}, \lambda \in \mathbb{C}$, então basta tomar

$$
v=i z_{a}
$$

Assim, $\boldsymbol{R e}\left\langle i z_{a}, z_{a}\right\rangle=0$ e, pelo Lema 2.0.7 o número

$$
\left\langle i z_{a}, \mathrm{i} \operatorname{grad} \log f\left(z_{a}\right)\right\rangle=\bar{\lambda}\left\|z_{a}\right\|^{2}
$$

tem argumento em valor absoluto menor que $\pi / 4$. Usando a continuidade da função

$$
z \longmapsto|\arg \langle i z, \mathrm{i} \operatorname{grad} \log f(z)\rangle|
$$

conseguimos uma vizinhança $U_{a}$ de $z_{a}$ tal que

$$
|\arg \langle i z, \mathrm{i} \operatorname{grad} \log f(z)\rangle|<\pi / 4
$$

para todo $z$ em $U_{a}$. Definimos então,

$$
v_{a}(z)=i z, \forall z \in U_{a}
$$


Desta maneira, cobrimos $S_{\varepsilon} \backslash K$ por abertos $U_{a}$ em cada qual está definido um campo de vetores tangentes satisfazendo as propriedades requeridas. Finalmente, utilizando-se uma partição diferenciável da unidade, obtemos um campo de vetores global $v(z)$. Tal campo será tangente a $S_{\varepsilon} \backslash K$ pois, para cada $z, v(z)$ é dado por uma combinação linear com coeficientes reais (fornecidos pela partição) de vetores tangentes. Precisamos porém, garantir que as "colagens"destes campos locais mantêm a propriedade

$$
|\arg \langle v(z), \operatorname{igrad} \log f(z)\rangle|<\pi / 4 \text {. }
$$

É o que faremos na proposição seguinte.

Proposição 2.0.12. Sejam $u, v_{a_{1}}, \ldots, v_{a_{n}}$ vetores de $\mathbb{C}^{n+1}$ tais que

$$
\left|\arg \left\langle v_{a_{i}}, u\right\rangle\right|<\pi / 4, i=1, \ldots, n \text {. }
$$

Então para toda combinação linear não nula, $v=\alpha_{1} v_{a_{1}}+\ldots+\alpha_{n} v_{a_{n}}$, com coeficientes reais, temos $|\arg \langle v, u\rangle|<\pi / 4$.

Demonstração. Mostraremos que o resultado é válido para $v=\alpha_{1} v_{a_{1}}+\alpha_{2} v_{a_{2}}$, o caso geral segue por indução. Considere

$$
\begin{aligned}
& \beta=\arg \langle v, u\rangle, \\
& \beta_{1}=\arg \left\langle\alpha_{1} v_{a_{1}}, u\right\rangle=\arg \left\langle v_{a_{1}}, u\right\rangle, \\
& \beta_{2}=\arg \left\langle\alpha_{2} v_{a_{2}}, u\right\rangle=\arg \left\langle v_{a_{2}}, u\right\rangle .
\end{aligned}
$$

Por hipótese, $\left|\beta_{1}\right|<\pi / 4$ e $\left|\beta_{2}\right|<\pi / 4$. Queremos mostrar que $|\beta|<\pi / 4$. Agora,

$$
\begin{aligned}
\left|\left\langle\alpha_{1} v_{\alpha 1}, u\right\rangle\right| \cos \beta_{1}+\left|\left\langle\alpha_{2} v_{\alpha 2}, u\right\rangle\right| \cos \beta_{2} & =\operatorname{Re}\left\langle\alpha_{1} v_{\alpha 1}, u\right\rangle+\operatorname{Re}\left\langle\alpha_{2} v_{\alpha 2}, u\right\rangle \\
& =\operatorname{Re}\left\langle\alpha_{1} v_{\alpha 1}+\alpha_{2} v_{\alpha 2}, u\right\rangle \\
& =\left|\left\langle\alpha_{1} v_{\alpha 1}+\alpha_{2} v_{\alpha 2}, u\right\rangle\right| \cos \beta
\end{aligned}
$$

Mas,

$$
\left|\left\langle\alpha_{1} v_{\alpha 1}+\alpha_{2} v_{\alpha 2}, u\right\rangle\right| \cos \beta \leq\left|\left\langle\alpha_{1} v_{\alpha 1}, u\right\rangle\right| \cos \beta+\left|\left\langle\alpha_{2} v_{\alpha 2}, u\right\rangle\right| \cos \beta
$$


Logo,

$$
\left|\left\langle\alpha_{1} v_{\alpha 1}, u\right\rangle\right|\left(\cos \beta_{1}-\cos \beta\right)+\left|\left\langle\alpha_{2} v_{\alpha 2}, u\right\rangle\right|\left(\cos \beta_{2}-\cos \beta\right) \leq 0 .
$$

Agora, suponha que $|\beta| \geq \pi / 4$. Então $\cos \beta$ pertence ao intervalo $\left[-1, \frac{\sqrt{2}}{2}\right]$. Como

$$
\cos \beta_{1}>\frac{\sqrt{2}}{2} \text { e } \cos \beta_{2}>\frac{\sqrt{2}}{2}
$$

segue que

$$
\left(\cos \beta_{1}-\cos \beta\right)>0 \quad \text { e } \quad\left(\cos \beta_{2}-\cos \beta\right)>0,
$$

o que contradiz a Equação 2.0.1 Portanto, $|\beta|<\frac{\pi}{4}$.

Isto conclui a demonstração do Lema 2.0.11.

Considere agora a normalização do campo $v(z)$ :

$$
w(z)=v(z) / \boldsymbol{\operatorname { R e }}\langle v(z), i \operatorname{grad} \log f(z)\rangle .
$$

Note que $w(z)$ também é diferenciável e tangente à $S_{\varepsilon} \backslash K$. Além disso, $w$ satisfaz as seguintes propriedades:

$$
\begin{aligned}
& \boldsymbol{\operatorname { R e }}\langle w(z), i \operatorname{grad} \log f(z)\rangle=1 \\
& |\boldsymbol{R e}\langle w(z), \operatorname{grad} \log f(z)\rangle|<1
\end{aligned}
$$

De fato, pelo Lema 2.0.11

$$
\begin{aligned}
& \boldsymbol{\operatorname { R e }}\langle w(z), i \operatorname{grad} \log f(z)\rangle=\frac{\boldsymbol{\operatorname { R e }}\langle v(z), i \operatorname{grad} \log f(z)\rangle}{\boldsymbol{\operatorname { R e }}\langle v(z), i \operatorname{grad} \log f(z)\rangle}=1 \\
& \boldsymbol{\operatorname { R e }}\langle w(z), i \operatorname{grad} \log f(z)\rangle=\left|\frac{\boldsymbol{\operatorname { R e }}\langle v(z), \operatorname{grad} \log f(z)\rangle}{\boldsymbol{\operatorname { R e }}\langle v(z), i \operatorname{grad} \log f(z)\rangle}\right|=\left|\frac{\operatorname{Im}\langle v(z), i \operatorname{grad} \log f(z)\rangle}{\operatorname{Re}\langle v(z), i \operatorname{grad} \log f(z)\rangle}\right|<1 .
\end{aligned}
$$

Em seguida, trabalharemos com as trajetórias da equação diferencial

$$
\frac{d z}{d t}=w(z)
$$


Lema 2.0.13. Dado $z_{0} \in S_{\varepsilon} \backslash K$, existe um único caminho diferenciável

$$
p: \mathbb{R} \longrightarrow S_{\epsilon}-K
$$

que satisfaz a equação diferencial $\frac{d p}{d t}(t)=w(p(t))$, com condição inicial $p(0)=z_{0}$.

Demonstração. Pelo Teorema de Existência e Unicidade de solução de uma equação diferencial, tal solução existe localmente e pode ser extendida a um intervalo maximal aberto de $\mathbb{R}$. Como $S_{\varepsilon} \backslash K$ não é compacto, para que seja possível extender a solução a toda reta real, deve-se assegurar que $p(t)$ não tende a $K$ quando $t$ tende a algum limite finito $t_{0}$. Isto é, devemos garantir que $|f(p(t))|$ não tende a zero, ou equivalentemente, que $\operatorname{Re} \log f(p(t))$ não tende a $-\infty$, pois $\log |f(p(t))|=\operatorname{Re} \log f(p(t))$. Mas, a derivada

$$
\frac{d}{d t}(\boldsymbol{R e} \log (f \circ p)(t))=\mathbf{R e}\left\langle\frac{d p}{d t}(t), \operatorname{grad} \log f(p(t))\right\rangle=\mathbf{R e}\langle w(p(t)), \operatorname{grad} \log f(p(t))\rangle
$$

tem valor absoluto menor que 1 . Portanto, Re $\log f(p(t))$ é limitada em cada vizinhança finita de $t$.

Escrevendo $\phi(z)=e^{i \theta(z)}$, segue da demonstração do Lema 2.0.2 e das propriedades do campo $w(z)$ que

$$
\frac{d}{d t}(\theta \circ p)(t)=\operatorname{Re}\left\langle\frac{d p}{d t}(t), i \operatorname{grad} \log f(p(t))\right\rangle
$$

é identicamente 1 , para todo $z=p(t)$ que é solução de $\frac{d z}{d t}=w(t)$. Portanto,

$$
\theta(p(t))=t+\text { constante }=t+\theta(p(0)) .
$$

Em outras palavras, $\phi$ projeta cada solução $p(t)$ em um caminho que percorre $S^{1}$ no sentido anti-horário com velocidade unitária.

Observação 2.0.14. Se $p(t)$ é um caminho na variedade $F_{\theta}$, então

$$
0=\frac{d}{d t}(\theta \circ p)(t)=\boldsymbol{R} e\left\langle\frac{d p}{d t}(t), i \operatorname{grad} \log f(p(t))\right\rangle .
$$


Isto nos diz que, segundo o produto interno real usual, o vetor $i \operatorname{grad} \log f(p(t))$ é ortogonal a $F_{\theta}$ em $z=p(t)$. Mas, então, a propriedade $\boldsymbol{R e}\langle w(z), i \operatorname{grad} \log f(z)\rangle=1$ diz que $w(z)$ tem componente não nula no espaço normal a $F_{\theta}$ em $z$, ou seja, as curvas integrais de $w(z)$ são transversais às variedades $F_{\theta}$.

A observação acima, juntamente com a descrição do comportamento da função $\theta$ ao longo de cada curva integral do campo $w(z), \theta(p(t))=t+\theta(p(0))$, sugerem como deve ser feita a "colagem"das fibras para obtermos a trivialização.

Consideremos o fluxo sobre $S_{\varepsilon} \backslash K$, gerado pelo campo $w$, isto é:

$$
\begin{aligned}
h:\left(S_{\varepsilon} \backslash K\right) \times \mathbb{R} & \longrightarrow S_{\varepsilon} \backslash K \\
(z, t) & \longmapsto p_{z}(t),
\end{aligned}
$$

em que $p_{z}: \mathbb{R} \longrightarrow S_{\varepsilon} \backslash K$ é a única curva integral com $p_{z}(0)=z$. Note que $h_{t}: S_{\varepsilon} \backslash K \longrightarrow$ $S_{\varepsilon} \backslash K$ dado por $h_{t}(z)=p_{z}(t)$ é um difeomorfismo para cada $t \in \mathbb{R}$. De fato, para cada $t \in \mathbb{R}$, a aplicação $h_{t}$ é diferenciável. Além disso, para $t_{1}$ e $t_{2}$ em $\mathbb{R}$, temos

$$
\begin{aligned}
\phi\left(h_{t_{1}+t_{2}}(z)\right) & =e^{i \theta\left(h_{t_{1}+t_{2}}(z)\right)}=e^{i \theta\left(p_{z}\left(t_{1}+t_{2}\right)\right)} \\
& =e^{i\left(\theta(z)+t_{1}+t_{2}\right)}=e^{i\left(\left(\theta(z)+t_{2}\right)+t_{1}\right)} \\
& =e^{i\left(\theta\left(p_{z}\left(t_{2}\right)\right)+t_{1}\right)}=e^{i \theta\left(p_{p_{z}\left(t_{2}\right)}\left(t_{1}\right)\right)} \\
& =e^{i \theta\left(h_{t_{1}}\left(p_{z}\left(t_{2}\right)\right)\right)}=e^{i \theta\left(h_{t_{1}}\left(h_{t_{2}}(z)\right)\right)} \\
& =e^{i \theta\left(h_{t_{1}} \circ h_{t_{2}}(z)\right)}=\phi\left(h_{t_{1}} \circ h_{t_{2}}(z)\right) .
\end{aligned}
$$

Como estamos trabalhando com uma determinação local de $\theta(z)$ então $\phi(z)$ é injetora. Portanto,

$$
h_{t_{1}} \circ h_{t_{2}}(z)=h_{t_{1}+t_{2}}(z)
$$

Em particular, $h_{t}^{-1}(z)=h_{-t}(z)$, de onde segue que $h_{t}$ é um difeomorfismo.

Observe também que cada fibra $F_{\theta}=\phi^{-1}\left(e^{i \theta}\right)$ é levada sobre a fibra $F_{\theta+t}$ por cada $h_{t}$. De fato, se $z \in F_{\theta}$, então

$$
\phi\left(h_{t}(z)\right)=\phi\left(p_{z}(t)\right)=e^{i \theta\left(p_{z}(t)\right)}=e^{i\left(t+\theta\left(p_{z}(0)\right)\right)}=e^{i(t+\theta(z))}=e^{i(t+\theta)},
$$


de onde segue que $h_{t}(z) \in F_{\theta+t}$. Como $h_{t}$ é difeomorfismo, segue a afirmação.

Após este longo trabalho, podemos enfim demonstrar o Teorema da Fibração:

Teorema 2.0.15 (O Teorema da Fibração). Seja $\varepsilon_{0}$ um raio de Milnor da aplicação $\phi$. Para $0<\varepsilon \leq \varepsilon_{0}$, o espaço $S_{\varepsilon} \backslash K$ é um fibrado diferenciável localmente trivial sobre $S^{1}$, com projeção $\phi(z)=\frac{f(z)}{|f(z)|}$.

Demonstração. Dado $e^{i \theta} \in S^{1}$, seja $U_{\theta}$ um intervalo aberto em $S^{1}$ da forma

$$
U_{\theta}=\left\{e^{i t} \in S^{1}: t \in J_{\theta}=(\theta-a, \theta+a), 0<a<\theta+\pi\right\}
$$

e considere a aplicação

$$
\begin{aligned}
\psi_{\theta}: U_{\theta} \times F_{\theta} & \longrightarrow \phi^{-1}\left(U_{\theta}\right) \\
\left(e^{i t}, z\right) & \longmapsto h_{t-\theta}(z) .
\end{aligned}
$$

Então,

$$
\left(\phi \circ \psi_{\theta}\right)\left(e^{i t}, z\right)=\phi\left(h_{t-\theta}(z)\right)=\phi\left(p_{z}(t-\theta)\right)=e^{i\left[(t-\theta)+\theta\left(p_{z}(0)\right)\right]}=e^{i(t-\theta+\theta(z))}=e^{i t},
$$

pois $z \in F_{\theta}$, isto é, $\theta(z)=\theta$. Em outras palavras, $\phi$ é uma projeção.

Precisamos mostrar ainda que $\psi_{\theta}$ é um difeomorfismo. Que $\psi_{\theta}$ é diferenciável, segue da composição de aplicações diferenciáveis:

$$
\begin{aligned}
& U_{\theta} \times F_{\theta} \longrightarrow J_{\theta} \times F_{\theta} \longrightarrow F_{\theta} \times(-a, a) \quad \longrightarrow \phi^{-1}\left(U_{\theta}\right) \\
& \left(e^{i t}, z\right) \longmapsto(t, z) \longmapsto(z, t-\theta) \longmapsto h(z, t-\theta) \text {, }
\end{aligned}
$$

pois $\psi_{\theta}\left(e^{i t}, z\right)=h(z, t-\theta)$.

Considerando a aplicação $g: \phi^{-1}\left(U_{\theta}\right) \longrightarrow U_{\theta} \times F_{\theta}$, dada por

$$
g(z)=\left(\phi(z), h_{\theta-\arg \phi(z)}(z)\right)=\left(e^{i \theta(z)}, h_{\theta-\theta(z)}(z)\right),
$$


obtemos,

$$
\psi_{\theta}(g(z))=\psi_{\theta}\left(e^{i \theta(z)}, h_{\theta-\theta(z)}(z)\right)=h_{\theta(z)-\theta}\left(h_{\theta-\theta(z)}(z)\right)=h_{0}(z)=p_{z}(0)=z .
$$

Isto é,

$$
\psi_{\theta} \circ g=I d_{\phi^{-1}\left(U_{\theta}\right)}
$$

Por outro lado,

$$
\begin{aligned}
g\left(\psi_{\theta}\left(e^{i t}, z\right)\right) & =g\left(h_{t-\theta}(z)\right) \\
& =\left(\phi\left(h_{t-\theta}(z)\right), h_{\theta-\arg \phi\left(h_{t-\theta}(z)\right)}\left(h_{t-\theta}(z)\right)\right) \\
& =\left(e^{i t}, h_{\theta-t}\left(h_{t-\theta}(z)\right)\right. \\
& =\left(e^{i t}, h_{0}(z)\right)=\left(e^{i t}, z\right) .
\end{aligned}
$$

Isto é,

$$
g \circ \psi_{\theta}=I d_{U_{\theta} \times F_{\theta}} .
$$

Portanto, $g=\psi_{\theta}^{-1}$. Para mostrarmos que $\psi_{\theta}^{-1}$ é diferenciável, basta considerar a aplicação

$$
z \longrightarrow(z, \arg \phi(z)) \longrightarrow(z, \theta-\arg \phi(z)) \longrightarrow h(z, \theta-\arg \phi(z))=h_{\theta-\arg (z)}(z),
$$

que é uma composição de aplicações diferenciáveis. Logo, as componentes $\phi(z)$ e $h_{\theta-\arg \phi(z)}(z)$ de $g(z)$ são diferenciáveis. Portanto, $g=\psi_{\theta}^{-1}$ é diferenciável. 


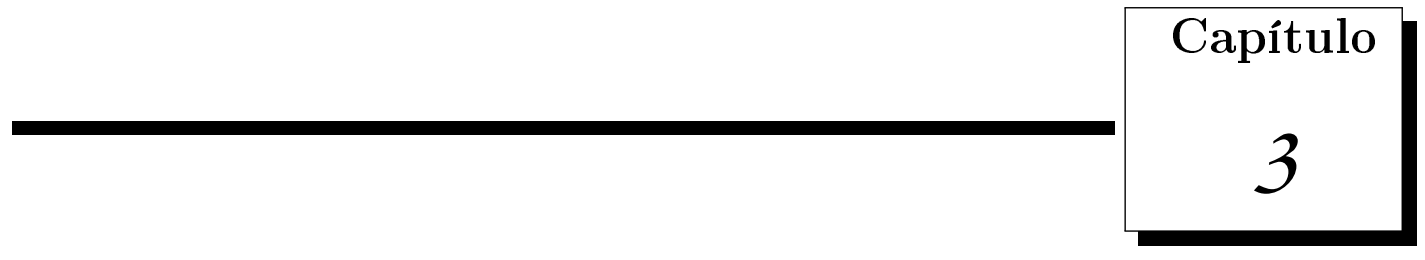

\section{A Topologia das Fibras}

Continuaremos a estudar a fibração localmente trivial, obtida no capítulo anterior,

$$
\phi: S_{\varepsilon} \backslash K \longrightarrow S^{1}
$$

associada a uma função polinomial complexa não nula $f\left(z_{1}, \ldots, z_{n+1}\right)$ que se anula na origem. Pelo Corolário 2.0.10 cada fibra $F_{\theta}=\phi^{-1}\left(e^{i \theta}\right)$ é uma variedade suave de dimensão real $2 n$. O principal resultado apresentado neste capítulo refere-se à topologia destas variedades e está descrito no seguinte teorema:

Teorema 3.0.16. Cada fibra $F_{\theta}$ tem o tipo de homotopia de um complexo $C W$ finito de dimensão $n$.

A demonstração do Teorema 3.0.16 dependerá de um estudo da teoria de Morse associada à função real diferenciável, $|f|$, restrita a $F_{\theta}$. Neste caso, será mostrado que o índice de Morse de $|f|$ em um ponto crítico é $\geq n$. Deste modo, nosso primeiro passo será identificar os pontos críticos desta aplicação.

Considere a aplicação diferenciável $a_{\theta}: F_{\theta} \longrightarrow \mathbb{R}$, definida por

$$
a_{\theta}(z)=\log |f(z)|
$$


Como a derivada da função real logarítmo é um isomorfismo, os pontos críticos da função $a_{\theta}$ são os mesmos de $|f|$ restrita a $F_{\theta}$. É conveniente trabalharmos com a função $a_{\theta}$ ao invés de $|f|$ devido à caracterização da derivada direcional , dada no capítulo anterior, que envolve a função $\log f(z)$.

Lema 3.0.17. Os pontos críticos de $a_{\theta}(z)=\log |f(z)|$ em $F_{\theta}$ são os pontos $z \in F_{\theta}$ para os quais o vetor grad $\log f(z)$ é um múltiplo complexo de z.

Demonstração. Escrevendo $f(z)=|f(z)| e^{i \arg f(z)}$ e considerando a equação

$$
\log f(z)=\log \left(|f(z)| e^{i \arg f(z)}\right)=\log |f(z)|+i \arg f(z),
$$

obtemos

$$
\operatorname{Re} \log f(z)=\log |f(z)|
$$

Derivando-se a função $\log |f(z)|=\boldsymbol{R e} \log f(z)$ ao longo de uma curva $z=p(t)$ em $\mathbb{C}^{n+1} \backslash V$, observamos que a derivada direcional na direção $v=\frac{d p}{d t}(t)$ é descrita por

$$
\frac{d}{d t} \mathbf{R e} \log f(p(t))=\mathbf{R e}\langle v, \operatorname{grad} \log f(p(t))\rangle
$$

basta aplicar a regra da cadeia e lembrar que $\frac{d}{d t}(f \circ p)(t)=d f(p(t)) \cdot \frac{d p}{d t}(t)$ e

$$
\operatorname{grad} \log f(p(t))=\frac{\operatorname{grad} f(p(t))}{\overline{f \circ p(t)}} .
$$

Assim, z será ponto crítico desta aplicação, restrita a $F_{\theta}$, se, e somente se, o vetor $\operatorname{grad} \log f(z)$ for normal a $F_{\theta}$ em $z$. (Aqui, "normal" significa ortogonal a todo vetor tangente, segundo o produto interno real).

Mas, segundo o produto interno real, $z$ é normal a $S_{\varepsilon}$ e, portanto, a $F_{\theta}$. Também, pela Observação 2.0.14 o vetor i grad $\log f(z)$ é normal a $F_{\theta}$ em $z$. Como $F_{\theta}$ tem codimensão real 2 e os vetores $z$ e i grad $\log f(z)$ são linearmente independentes sobre $\mathbb{R}$ (Lema 2.0.6), conclui-se que os vetores $z$ e i grad $\log f(z)$ geram o espaço normal a $F_{\theta}$ em $z$. Deste 
modo, $z$ será ponto crítico de $a_{\theta}$ se, e somente se, existirem $\lambda_{1}, \lambda_{2} \in \mathbb{R}$ de maneira que

$$
\operatorname{grad} \log f(z)=\lambda_{1} z+\lambda_{2} \text { i grad } \log f(z),
$$

isto é,

$$
\operatorname{grad} \log f(z)=\frac{\lambda_{1}}{1-\lambda_{2} i} z
$$

Observação 3.0.18. O espaço tangente à $F_{\theta}$ em um ponto crítico $z$ de $a_{\theta}$ é, de fato, o subespaço vetorial complexo de $\mathbb{C}^{n+1}$ constituído de todos os vetores $v \in \mathbb{C}^{n+1}$ tais que $\langle v, z\rangle=0$. Isto segue do fato que, se $i$ grad $\log f(z)$ for um múltiplo complexo de $z$, então um vetor $v$ será real ortogonal a ambos z e $i \operatorname{grad} \log f(z)$ se, e somente se, ele for complexo ortogonal a z. Com efeito, temos:

$$
\begin{gathered}
\left\{\begin{array} { l } 
{ \boldsymbol { R } \boldsymbol { e } \langle v , z \rangle = 0 } \\
{ \boldsymbol { R } e \langle v , i \operatorname { g r a d } \operatorname { l o g } f ( z ) \rangle = 0 }
\end{array} \Longleftrightarrow \left\{\begin{array}{l}
\boldsymbol{R} \boldsymbol{e}\langle v, z\rangle=0 \\
\boldsymbol{R} e\left\langle v,\left(\lambda_{1}+i \lambda_{2}\right) z\right\rangle=0 ; \lambda_{1}, \lambda_{2} \in \mathbb{R} ;
\end{array}\right.\right. \\
\Longleftrightarrow\left\{\begin{array} { l } 
{ \boldsymbol { R } \boldsymbol { x } \langle v , z \rangle = 0 } \\
{ \boldsymbol { R } e \langle v , \lambda _ { 1 } z \rangle + \boldsymbol { R } \boldsymbol { e } \langle v , i \lambda _ { 2 } z \rangle = 0 }
\end{array} \Longleftrightarrow \left\{\begin{array}{l}
\boldsymbol{R} \boldsymbol{e}\langle v, z\rangle=0 \\
\boldsymbol{R} e\left\langle v, i \lambda_{2} z\right\rangle=0 ;
\end{array}\right.\right. \\
\Longleftrightarrow\left\{\begin{array}{l}
\boldsymbol{R} e\langle v, z\rangle=0 \\
\boldsymbol{I m}\langle v, z\rangle=0,
\end{array}, \text { isto é, }\langle v, z\rangle=0 .\right.
\end{gathered}
$$

Estudaremos agora, a Hessiana de $a_{\theta}$ em um ponto crítico, de modo a computar seu índice de Morse. Usaremos a seguinte interpretação da Hessiana: Dado um vetor $v$ tangente a $F_{\theta}$ em um ponto crítico $z$, escolheremos um caminho diferenciável $p: \mathbb{R} \longrightarrow F_{\theta}$ com vetor velocidade $\frac{d p}{d t}=v$ em $p(0)=z$. Então a derivada segunda

$$
\ddot{a}_{\theta}(t)=\frac{d^{2}}{d t^{2}} a_{\theta}(p(t))
$$

em $\mathrm{t}=0$ poderá ser expressa como uma função quadrática. Tal função quadrática será a Hessiana de $a_{\theta}$. 
Lema 3.0.19. Com a notação anterior, a derivada segunda de $a_{\theta}(p(t))$ em $t=0$ é dada por uma expressão da forma

$$
\ddot{a}_{\theta}=\sum \boldsymbol{R} \boldsymbol{e}\left(b_{j k} v_{j} v_{k}\right)-c\|v\|^{2}
$$

em que $\left(b_{j k}\right)$ é uma matriz de números complexos e c é um número real positivo.

Demonstração. Note primeiramente, que o caminho $p(t)$ está contido na variedade $F_{\theta}$. Portanto, $\frac{f}{|f|}=e^{i \theta}$ é constante ao longo deste caminho. Derivando-se a identidade

$$
a_{\theta}(p(t))=\log |f(p(t))|=\log f(p(t))-i \theta
$$

obtemos

$$
\dot{a_{\theta}}=\frac{d}{d t} \log f=\sum_{j=1}^{n+1}\left(\frac{\partial \log f}{\partial z_{j}}\right) \frac{d p_{j}}{d t} .
$$

Derivando-se mais uma vez,

$$
\ddot{a_{\theta}}=\sum_{j=1}^{n+1}\left(\frac{\partial \log f}{\partial z_{j}}\right) \frac{d^{2} p_{j}}{d t^{2}}+\sum_{j, k=1}^{n+1}\left(\frac{\partial^{2} \log f}{\partial z_{j} \partial z_{k}}\right) \frac{d p_{j}}{d t} \frac{d p_{k}}{d t} .
$$

Escolhendo $t=0, \operatorname{grad} \log f(z)=\lambda z, \lambda \in \mathbb{C}$ (pelo Lema 3.0.17), e usando a notação

$$
D_{j k}=\frac{\partial^{2} \log f}{\partial z_{j} \partial z_{k}}
$$

podemos reescrever

$$
\ddot{a_{\theta}}=\langle\ddot{p}, \lambda z\rangle+\sum_{j, k=1}^{n+1} D_{j k} v_{j} v_{k}
$$

em que o lado esquerdo da equação é real, pois é a segunda derivada de uma função real. Agora, multiplicando-se os dois lados da equação por $\lambda$ e tomando-se a parte real, obtemos:

$$
\ddot{a_{\theta}} \mathbf{R e}(\lambda)=|\lambda|^{2} \mathbf{R e}\langle\ddot{p}, z\rangle+\sum_{j, k=1}^{n+1} \mathbf{R e}\left(\lambda D_{j k} v_{j} v_{k}\right) .
$$


Substituindo-se a identidade $\operatorname{Re}\langle\ddot{p}, z\rangle=-\|v\|^{2}$, obtida derivando-se $\langle p(t), p(t)\rangle=\varepsilon^{2}$ duas vezes, encontramos

$$
\ddot{a_{\theta}} \mathbf{R e}(\lambda)=\sum_{j, k=1}^{n+1} \boldsymbol{\operatorname { R e }}\left(\lambda D_{j, k} v_{j} v_{k}\right)-\|\lambda v\|^{2} .
$$

Pelo Lema 2.0.7 temos $\boldsymbol{R e}(\lambda)>0$. Então, dividindo-se a equação por $\boldsymbol{R e}(\lambda)$, concluímos a demonstração.

Podemos avaliar agora o índice de Morse da função $a_{\theta}$.

Lema 3.0.20. O indice de Morse de $a_{\theta}: F_{\theta} \longrightarrow \mathbb{R}$ em um ponto crítico é $\geq n$.

Demonstração. Como vimos no Capítulo 1, o índice de Morse, I, da função quadrática

$$
H(v)=\operatorname{Re}\left(\sum_{j, k=1}^{n+1} b_{j k} v_{j} v_{k}\right)-c\|v\|^{2}
$$

com $v$ variando em $T_{z} F_{\theta}$, é definido como sendo a dimensão máxima dentre os subespaços vetoriais de $T_{z} F_{\theta}$, nos quais $H$ é definida negativa.

Se $H(v) \geq 0$ para algum vetor $v$, não nulo, então $H(i v)<0$, pois o primeiro termo na expressão para $H(v)$ muda de sinal, enquanto o segundo termo permanece negativo. Note que, pela Observação 3.0.18, o vetor $i v$ também está em $T_{z} F_{\theta}$.

Consideremos a decomposição do espaço tangente $T_{z} F_{\theta}$ em uma soma direta real

$$
T_{z} F_{\theta}=T_{0} \oplus T_{1}
$$

na qual a Hessiana é definida negativa em $T_{0}$ e definida semi-positiva e $T_{1}$. Deste modo, a dimensão de $T_{0}$ é igual ao índice de Morse, I, de $H$.

Por outro lado, $H$ também é definida negativa em $i T_{1}$. Logo,

$$
I \geq \operatorname{dim}\left(i T_{1}\right)=\operatorname{dim} T_{1}=\operatorname{dim} T_{z} F_{\theta}-\operatorname{dim} T_{0}=2 n-I .
$$

Portanto, $I \geq n$.

O próximo passo é verificar que os pontos críticos situam-se todos em um subconjunto compacto de $F_{\theta}$. 
Lema 3.0.21. Existe uma constante real $\eta_{\theta}>0$ tal que, se $z \in F_{\theta}$ for ponto crítico de $a_{\theta}$, então $|f(z)| \geq \eta_{\theta}$. Isto é, os pontos críticos de $a_{\theta}$ estão todos no subconjunto compacto

$$
\mathrm{C}_{\theta}=\left\{z \in F_{\theta} ;|f(z)| \geq \eta_{\theta}\right\}
$$

Demonstração. Suponha que existam pontos críticos z de $a_{\theta}=\log |f|$, com $|f(z)|$ arbitrariamente próximos de zero. Então estes pontos críticos têm um ponto limite $z_{0}$ no conjunto compacto $S_{\varepsilon}$, de maneira que $|f(z)| \longrightarrow\left|f\left(z_{0}\right)\right|=0$. Assim, se encontrarmos uma curva diferenciável $p:(0, \delta) \rightarrow F_{\theta}$, com $p(t) \longrightarrow z_{0}$ quando $t \longrightarrow 0$, ao longo da qual $|f|$ não pode tender a zero, chegaremos a uma contradição.

Seja

$$
W=\left\{z \in \mathbb{C}^{n+1} ; \operatorname{grad} f(z)=\lambda z, \lambda \in \mathbb{C}\right\}
$$

Vimos na demonstração do Lema 2.0.7 que $W$ é um conjunto algébrico real.

Considere também,

$$
B_{\theta}=\left\{z \in \mathbb{C}^{n+1} ; \boldsymbol{\operatorname { R e }}\left(i e^{-i \theta} f(z)\right)=0\right\}
$$

que é um conjunto algébrico real, já que $f$ é polinomial. Considere por fim, o conjunto semi-algébrico real

$$
U_{\theta}=\left\{z \in \mathbb{C}^{n+1} ; \operatorname{Im}\left(i e^{-i \theta} f(z)\right)>0\right\}
$$

Observe que, $z \in U_{\theta} \cap B_{\theta}$ se, e somente se, $\arg f(z)=\theta$.

Estamos supondo então, que existem pontos $z$ arbitrariamente próximos de $z_{0}$ com

$$
z \in\left(\left(S_{\varepsilon} \cap W \cap B_{\theta}\right) \cap U_{\theta}\right) \subset F_{\theta} .
$$

Como $S_{\varepsilon}$ também é um conjunto algébrico real, pelo Lema de Seleção de Curva, existe uma curva diferenciável $p:(0, \delta) \longrightarrow F_{\theta}$, com $p(t) \in W \cap B_{\theta} \cap U_{\theta}$ e com $p(t) \rightarrow z_{0}$ quando $t \rightarrow 0$. Então, usando o fato de $p(t)$ pertencer a $W$ e o Lema 3.0.17 observamos que $p(t)$ é constituído de pontos críticos de $a_{\theta}$. Deste modo, a função $a_{\theta}$, e portanto $|\mathbf{f}|$, é constante 
ao longo desta curva. Como $p(t) \in F_{\theta},|f(p(t))|$ deve ser constante não nula. De onde segue a contradição.

Mostremos por fim que o conjunto $C_{\theta}$ é, de fato, compacto. Considere o subconjunto fechado de $\mathbb{C}^{n+1}$

$$
A_{\theta}=\left\{z \in \mathbb{C}^{n+1} ;|f(z)| \geq \eta_{\theta}\right\}
$$

Então,

$$
A_{\theta} \cap\left(S_{\varepsilon} \backslash K\right)=A_{\theta} \cap S_{\varepsilon} \quad \text { e } \quad C_{\theta}=A_{\theta} \cap F_{\theta} .
$$

Como $\phi^{-1}\left(e^{i \theta}\right)=F_{\theta}$ é fechado em $S_{\varepsilon} \backslash K$, então $F_{\theta}=F \cap\left(S_{\varepsilon} \backslash K\right)$, para algum $F$ fechado em $\mathbb{C}^{n+1}$. Assim,

$$
C_{\theta}=A_{\theta} \cap F_{\theta}=A_{\theta} \cap F \cap\left(S_{\varepsilon} \backslash K\right)=A_{\theta} \cap F \cap S_{\varepsilon},
$$

ou seja, $C_{\theta}$ é um subconjunto fechado de $S_{\varepsilon}$ que é compacto em $\mathbb{C}^{n+1}$, portanto $C_{\theta}$ é compacto em $S_{\varepsilon}$ e, consequentemete, em $F_{\theta}$.

Lema 3.0.22. Existe uma aplicação diferenciável

$$
s_{\theta}: F_{\theta} \longrightarrow \mathbb{R}_{+}
$$

tal que os pontos críticos de $s_{\theta}$ são não degenerados, com indice de Morse $\geq n$ e tal que

$$
s_{\theta}(z)=|f(z)|
$$

para $|f(z)|$ suficientemente próximo de zero.

Demonstração. Segue do fato que as funções de Morse formam um aberto denso em $C^{\infty}\left(\mathrm{F}_{\theta}, \mathbb{R}\right)$ com a topologia de Whitney: Segundo Morse [20], Teorema 8.7, pág. 178, podemos escolher uma função $s_{\theta}$ que coincida com $|f|$ exceto em uma vizinhança compacta do conjunto crítico, de maneira que $s_{\theta}$ tenha apenas pontos críticos não degenerados e suas derivadas primeira e segunda se aproximem uniformemente das derivadas primeira e segunda de $|f|$ sobre qualquer vizinhança compacta de $F_{\theta}$. Como todos os pontos críticos 
de $|f|$ têm índice $\geq n$, segue que se a aproximação for suficientemente próxima, os pontos críticos de $s_{\theta}$ também terão índice $\geq n$ (veja [18], 22.4). Isto completa a demonstração.

Observação 3.0.23. Como os pontos críticos de $s_{\theta}$ são não degenerados, eles são isolados. Além disso, os pontos críticos de $s_{\theta}$ estão contidos em um compacto. Portanto, $s_{\theta}$ tem somente um número finito de pontos críticos.

Agora podemos provar o Teorema 3.0.16

Demonstração do Teorema 3.0.16 Para aplicarmos a teoria de Morse na sua forma usual, é necessário considerar uma aplicação não degenerada $g_{\theta}: F_{\theta} \longrightarrow \mathbb{R}$ com a propriedade: o conjunto dos $z$ em $F_{\theta} \operatorname{com} g_{\theta}(z) \leq c$ é compacto, para toda constante real $c$. Em outras palavras, a aplicação $g_{\theta}$ deve ser própria e limitada inferiormente.

Afirmamos que a função

$$
g_{\theta}(z)=-\log s_{\theta}(z)
$$

satisfaz esta condição. Ou seja,

$$
g_{\theta}^{-1}(-\infty, c]=s_{\theta}^{-1}\left[e^{-c}, \infty\right)
$$

é compacto para cada $c$.

De fato, seja $M_{\theta}$ uma vizinhança compacta dos pontos críticos de $|f|$, fornecida pelo Lema 3.0.22 fora da qual $|f|=s_{\theta}$. Com um argumento análogo ao dado para garantir a compacidade de $C_{\theta}$ no Lema 3.0 .21 concluímos que o conjunto

$$
\left\{z \in F_{\theta} ;|f(z)| \geq e^{-c}\right\}
$$

é compacto para cada $c$. Como $s_{\theta}(z)=|f(z)|$ fora de $M_{\theta}$, segue que:

$$
s_{\theta}^{-1}\left[e^{-c}, \infty\right) \subset\left(|f| / F_{\theta}\right)^{-1}\left[e^{-c}, \infty\right) \cup M_{\theta},
$$

para cada c, ou seja, $s_{\theta}^{-1}\left[e^{-c}, \infty\right)$ é um fechado em $F_{\theta}$ contido em um subconjunto compacto de $F_{\theta}$, portanto é compacto em $F_{\theta}$. 
Como o índice de Morse $I$ de $s_{\theta}$ ou de $\log s_{\theta}$ em um ponto crítico $z$ é maior ou igual a $n$, o índice de Morse de $-\log s_{\theta}$ é

$$
\tilde{I}_{\theta}=\operatorname{dim} T_{z} F_{\theta}-I=2 n-I \leq n
$$

Agora, de acordo com o principal teorema da teoria de Morse [18], a variedade $F_{\theta}$ tem o tipo de homotopia de um complexo $C W$ de dimensão $\leq n$, obtido pela adjunção de uma célula de dimensão $\tilde{I}_{\theta}$ para cada ponto crítico de índice $\tilde{I}_{\theta} \leq n$. Como existe apenas um número finito de pontos críticos, o complexo $C W$ é finito. Isto demonstra o teorema. 



-

\section{A Topologia das Fibras para o caso de} um ponto crítico isolado

Neste capítulo consideraremos a hipótese adicional de que a função polinomial complexa $f\left(z_{1}, \ldots, z_{n+1}\right)$ não tem pontos críticos em uma vizinhança da origem, exceto possivelmente na própria origem. Isto é, a origem será um ponto não singular ou um ponto singular isolado da hipersuperfície $V=f^{-1}(0)$. Vamos supor também que $n \geq 1$.

Seja $S_{\varepsilon}$ contida em uma vizinhança da origem, nas condições acima. Então, o vetor $\operatorname{grad} f(z)$ é não nulo para todo $z \neq 0$ nessa vizinhança, em particular para $z \in S_{\varepsilon}$.

Lema 4.0.24. Os pontos críticos de $\left.f\right|_{S_{\varepsilon}}$ são os pontos $z$ em $S_{\varepsilon}$ para os quais o vetor grad $f(z)$ é um múltiplo complexo de z.

Demonstração. Como $T_{z} S_{\varepsilon}$ é um espaço vetorial real, consideramos a identificação natural de $\mathbb{C}$ com $\mathbb{R}^{2}$ e teremos $z \in S_{\varepsilon}$ ponto crítico de $\left.f\right|_{S_{\varepsilon}}$ se, e somente se, o núcleo da derivada

$$
\begin{aligned}
& d\left(\left.f\right|_{S_{\varepsilon}}\right)(z)=\left.d f(z)\right|_{T_{z} S_{\varepsilon}}: T_{z} S_{\varepsilon} \quad \longrightarrow \quad \mathbb{R}^{2} \\
& v \quad \longmapsto\langle v, \operatorname{grad} f(z)\rangle
\end{aligned}
$$

tem dimensão maior ou igual a $2 n$, já que $T_{z} S_{\varepsilon}$ tem dimensão $2 n+1$. (Note que se considerássemos $\mathrm{n}=0$, então todo $z$ em $S_{\varepsilon}$ seria crítico de $\left.f\right|_{S_{\varepsilon}}$.) 
Suponhamos que $z$ e grad $f(z)$ sejam linearmente independentes sobre $\mathbb{C}$. Afirmamos que $z$ não é crítico de $\left.f\right|_{S_{\varepsilon}}$. De fato, se $z$ e grad $f(z)$ são linearmente independentes sobre $\mathbb{C}$ então o sistema

$$
\left\{\begin{array}{l}
\langle v, z\rangle=0 \\
\langle v, \operatorname{grad} f(z)\rangle=1
\end{array}\right.
$$

tem solução $v \neq 0$. A primeira equação fornece $\operatorname{Re}\langle v, z\rangle=0$, ou seja, $v \in T_{z} S_{\varepsilon}$. Mas, $\langle i v, z\rangle=i\langle v, z\rangle=0$. Portanto, $i v$ também pertence a $T_{z} S_{\varepsilon}$ e $\langle i v, \operatorname{grad} f(z)\rangle=i$, pela segunda equação. Como os vetores $v$ e $i v$ são linearmente independentes sobre $\mathbb{R}$, então o núcleo de $d\left(\left.f\right|_{S_{\varepsilon}}\right)(z)$ tem dimensão menor ou igual a $(2 n+1)-2=2 n-1$, de modo que $z$ não é crítico.

Por outro lado, se grad $f(z)=\lambda z$, para algum $\lambda$ em $\mathbb{C}$. Então,

$$
\begin{aligned}
\operatorname{ker}\left(d\left(\left.f\right|_{S_{\varepsilon}}\right)(z)\right) & =\left\{v \in T_{z} S_{\varepsilon} ;\langle v, \operatorname{grad} f(z)\rangle=\langle v, \lambda z\rangle=0\right\} \\
& =\left\{v \in \mathbb{C}^{n+1} ; \operatorname{Re}\langle v, z\rangle=0,\langle v, \lambda z\rangle=0\right\} \\
& =\left\{v \in \mathbb{C}^{n+1} ;\langle v, z\rangle=0\right\}
\end{aligned}
$$

que é um espaço vetorial real de dimensão $2 n$. Logo, z é crítico. Isto completa a demonstração.

Lema 4.0.25. Para $\varepsilon>0$ suficientemente pequeno, $K=S_{\varepsilon} \cap V$ não contém pontos críticos de $\left.f\right|_{S_{\varepsilon}}$.

Demonstração. A demonstração é baseada no Lema de Seleção da Curva 2.0.8.

Sejam os conjuntos algébricos reais

$$
V=f^{-1}(0) \quad \text { e } \quad W=\left\{z \in \mathbb{C}^{n+1} ; \operatorname{grad} f(z)=\lambda z, \lambda \in \mathbb{C}\right\}
$$

e seja $U$ o conjunto semi-algébrico real,

$$
U=\left\{z \in \mathbb{C}^{n+1} ;\langle z, z\rangle=\|z\|^{2}>0\right\}=\mathbb{C}^{n+1} \backslash\{0\}
$$

Suponhamos, por absurdo, que para todo $\varepsilon>0$ exista $z \in V$, crítico de $\left.f\right|_{S_{\varepsilon}}$. Então $0 \in \overline{V \cap W \cap U}$ e, portanto, pelo Lema de Seleção da Curva, existe uma curva analítica 
real

$$
p:\left[0, \delta^{\prime}\right) \longrightarrow \mathbb{C}^{n+1}
$$

$\operatorname{com} p(0)=0$ e $p(t) \in V \cap W \cap U$ para $t>0$.

Agora, como $p(t) \in V$, temos $f(p(t))=0$ para $t \in\left[0, \delta^{\prime}\right)$, de onde segue que

$$
\left\langle\frac{d p}{d t}(t), \operatorname{grad} f(p(t))\right\rangle=\frac{d}{d t}(f \circ p)(t) \equiv 0
$$

De $p(t) \in W$, temos grad $f(p(t))=\lambda p(t)$ para algum $\lambda \in \mathbb{C}$. Mas, então,

$$
0=\left\langle\frac{d p}{d t}(t), \operatorname{grad} f(p(t))\right\rangle=\left\langle\frac{d p}{d t}(t), \lambda p(t)\right\rangle
$$

$\log \mathrm{O}$

$$
0=2 \boldsymbol{R e}\left\langle\frac{d p}{d t}(t), p(t)\right\rangle=\frac{d}{d t}\|p(t)\|^{2} .
$$

Em outras palavras, $p(t)$ é constante e, como $p(0)=0$, devemos ter $p(t) \equiv 0$, contrariando o fato de, por construção, $p(t)$ pertencer a $U$, para $t>0$. Esta contradição demonstra o Lema 4.0.25

Lema 4.0.26. Para $\varepsilon>0$ suficientemente pequeno, o fecho em $S_{\varepsilon}$ de cada fibra $F_{\theta}$ é uma variedade diferenciável de dimensão real $2 n$, com bordo. O interior dessa variedade é $F_{\theta}$ e o bordo é precisamente $K$.

Demonstração. Devemos verificar, primeiramente, que os pontos de $K$ pertencem ao fecho de $F_{\theta}$ em $S_{\varepsilon}$.

Dado $z_{0} \in K$, como $z_{0}$ não é crítico de $\left.f\right|_{S_{\varepsilon}}$, existem, pela forma local das submersões, coordenadas reais $u_{1}, \ldots, u_{2 n+1}$ em uma vizinhança $U^{\prime}$ de $z_{0}$ tal que

$$
f(z)=u_{1}(z)+i u_{2}(z)
$$

para todo $z$ em $U^{\prime}$. Assim, se $U^{\prime \prime}$ é uma vizinhança qualquer de $z_{0}$ em $S_{\varepsilon}$, para todo $z$ em $U=U^{\prime} \cap U^{\prime \prime}, f(z)$ também se escreve como $f(z)=u_{1}(z)+i u_{2}(z)$. Observe que $U$ não está inteiramente contido em $K$, já que $z_{0}$ não é crítico de $\left.f\right|_{S_{\varepsilon}}$, e como 
$0=f\left(z_{0}\right)=u_{1}\left(z_{0}\right)+i u_{2}\left(z_{0}\right)$, a vizinhança $U$ contém pontos $z$ tais que

$$
\left(u_{1}(z)\right)^{2}+\left(u_{2}(z)\right)^{2} \neq 0 \quad \text { e } \arg \left(u_{1}(z)+i u_{2}(z)\right)=\theta
$$

para cada $\theta$. Segue então que $z_{0} \in \overline{F_{\theta}}$.

Para concluir a demonstração observemos que

$$
\overline{F_{\theta}} \cap U=\left(F_{\theta} \cup K\right) \cap U=\left(F_{\theta} \cup\left\{z ; u_{1}(z)=u_{2}(z)=0\right\}\right) \cap U
$$

e que um ponto $z$ pertence a $F_{\theta} \cap U$ se, e somente se, $\arg \left(u_{1}(z)+i u_{2}(z)\right)=\theta$ (portanto $u_{1}(z) \neq 0$ ou $\left.u_{2}(z) \neq 0\right)$. Em outras palavras, os pontos de $F_{\theta} \cap U$ não pertencem ao bordo de $\overline{F_{\theta} \cap U}$. Como todo ponto de $K$ admite uma tal vizinhança $U$, segue o resultado.

Corolário 4.0.27. A variedade compacta com bordo, $\overline{F_{\theta}}$, está mergulhada em $S_{\varepsilon}$ de tal modo que tem o mesmo tipo de homotopia que seu complemento $S_{\varepsilon} \backslash \overline{F_{\theta}}$.

Demonstração. Como $\overline{F_{\theta}}$ é uma subvariedade de $S_{\varepsilon}$, a inclusão $i: \overline{F_{\theta}} \longrightarrow S_{\varepsilon}$ é um mergulho. Agora, o Teorema da Fibração 2.0 .15 fornece a fibração localmente trivial $\phi: S_{\varepsilon} \backslash K \longrightarrow S^{1}$. Segue que a restrição

$$
\phi: S_{\varepsilon} \backslash\left(K \cup \phi^{-1}\left(e^{i \theta}\right)\right) \longrightarrow S^{1} \backslash\left\{e^{i \theta}\right\}
$$

é uma fibração localmente trivial. Mas, $S^{1} \backslash\left\{e^{i \theta}\right\}$ é contrátil. Portanto, a fibração é (globalmente) trivial, ou seja,

$$
S_{\varepsilon} \backslash \overline{F_{\theta}}=S_{\varepsilon} \backslash\left(K \cup F_{\theta}\right) \text { é difeomorfo a }\left(S^{1} \backslash\left\{e^{i \theta}\right\}\right) \times F_{\theta^{\prime}},
$$

em que $F_{\theta^{\prime}}$ é qualquer fibra diferente de $F_{\theta}$. Usando novamente a contratibilidade de $S^{1} \backslash\left\{e^{i \theta}\right\}, S_{\varepsilon} \backslash \overline{F_{\theta}}$ tem $F_{\theta^{\prime}}$ como um retrato por deformação. Como as fibras são difeomorfas, temos

$$
S_{\varepsilon} \backslash \overline{F_{\theta}} \sim F_{\theta^{\prime}} \sim F_{\theta}
$$

em que $\sim$ significa "tem o mesmo tipo de homotopia que". 
Como o interior de $\overline{F_{\theta}}$ é justamente $F_{\theta}$, segue que $F_{\theta}$ tem o mesmo tipo de homotopia que $\overline{F_{\theta}}$.

Corolário 4.0.28. A homologia de $F_{\theta}$ está concentrada nas dimensões 0 e n. Além disso, $H_{0}\left(F_{\theta}\right) \cong \mathbb{Z}$.

Demonstração. Pelo Teorema 3.0.16 temos $H_{q}\left(F_{\theta}\right)=0$ para $q>n$. Também,

$$
H_{q}\left(F_{\theta}\right) \cong H_{q}\left(S_{\varepsilon} \backslash \overline{F_{\theta}}\right)
$$

já que $F_{\theta}$ é difeomorfa a um retrato por deformação de $S_{\varepsilon} \backslash \overline{F_{\theta}}$. Portanto, basta considerarmos $H_{q}\left(S_{\varepsilon} \backslash \overline{F_{\theta}}\right)$. O Teorema da dualidade de Alexander (veja [21] pág. 424) fornece um isomorfismo

$$
H^{2 n+1-q}\left(\overline{F_{\theta}}\right) \cong H_{q}\left(S_{\varepsilon}, S_{\varepsilon} \backslash \overline{F_{\theta}}\right)
$$

Assim, da sequência exata de homologia do par $\left(S_{\varepsilon}, S_{\varepsilon} \backslash \overline{F_{\theta}}\right)$,

$$
\ldots \longrightarrow H_{q}\left(S_{\varepsilon}\right) \longrightarrow H_{q}\left(S_{\varepsilon}, S_{\varepsilon} \backslash \overline{F_{\theta}}\right) \longrightarrow H_{q-1}\left(S_{\varepsilon} \backslash \overline{F_{\theta}}\right) \longrightarrow H_{q-1}\left(S_{\varepsilon}\right) \longrightarrow \ldots
$$

temos, para $2 \leq q \leq n$,

$$
H_{q-1}\left(S_{\varepsilon} \backslash \overline{F_{\theta}}\right) \cong H_{q}\left(S_{\varepsilon}, S_{\varepsilon} \backslash \overline{F_{\theta}}\right) \cong H^{2 n+1-q}\left(\overline{F_{\theta}}\right)
$$

Agora, pelo Teorema 3.0.16 para $2 n+1-q>n$, temos

$$
H^{2 n+1-q}\left(\overline{F_{\theta}}\right)=0 \text {. }
$$

Portanto,

$$
H_{q-1}\left(F_{\theta}\right) \cong H_{q-1}\left(S_{\varepsilon} \backslash \overline{F_{\theta}}\right)=0
$$

para $q<n+1$.

Além disso,

$$
\left.\left.\ldots \longrightarrow H_{2 n+1}\left(S_{\varepsilon} \backslash \overline{F_{\theta}}\right)\right) \longrightarrow H_{2 n+1}\left(S_{\varepsilon}\right) \longrightarrow H_{2 n+1}\left(S_{\varepsilon}, S_{\varepsilon} \backslash \overline{F_{\theta}}\right) \longrightarrow H_{2 n}\left(S_{\varepsilon} \backslash \overline{F_{\theta}}\right)\right) \longrightarrow,
$$


$\left.\operatorname{com} H_{2 n+1}\left(S_{\varepsilon} \backslash \overline{F_{\theta}}\right)\right)=0, H_{2 n+1}\left(S_{\varepsilon}\right)=\mathbb{Z}$ e $H_{2 n}\left(S_{\varepsilon} \backslash \overline{F_{\theta}}\right)=0$. Portanto,

$$
\left.H^{0}\left(\overline{F_{\theta}}\right) \cong H_{2 n+1}\left(S_{\varepsilon}, S_{\varepsilon} \backslash \overline{F_{\theta}}\right)\right) \cong \mathbb{Z}
$$

ou seja, $F_{\theta}$ é conexa.

Lema 4.0.29. A fibra $F_{\theta}$ é $(n-1)$-conexa.

Demonstração. Pelo corolário anterior, temos $H_{i}\left(F_{\theta}\right)=0$ para $1 \leq i<n$. Então, se mostrarmos que $F_{\theta}$ é simplesmente conexa para $n \geq 2$, o resultado seguirá do Teorema de Hurewicz [25]. Agora, também pelo corolário anterior, $F_{\theta}$ é conexa e, como é localmente conexa por caminhos, segue que é conexa por caminhos. Assim, é suficiente mostrarmos que $\pi_{1}\left(F_{\theta}\right)=0$ para $n \geq 2$.

Consideremos a função

$$
-s_{\theta}: \overline{F_{\theta}} \longrightarrow \mathbb{R}
$$

em que $s_{\theta}$ é a função de Morse obtida no Lema 3.0.22. Então, $-s_{\theta}$ é uma função de Morse em $\overline{F_{\theta}}$ cujos pontos críticos têm índice $I \leq n$. Notando que o índice $I$ de $-s_{\theta}$ associado ao menor valor crítico é zero, concluímos que $\overline{F_{\theta}}$ é obtida de um disco $D_{0}^{2 n}$ pela adjunção de células de dimensão menor ou igual a $n$. Agora, $S_{\varepsilon} \backslash D_{0}^{2 n}$ é contrátil e, portanto, simplesmente conexo. Também, a adjunção de uma célula $e_{\lambda}$ de dimensão $\lambda<n$ a $D_{0}^{2 n}$, não altera o grupo fundamental de $S_{\varepsilon} \backslash\left(D_{0}^{2 n} \cup e_{\lambda}\right)$, desde que $\lambda \leq\left(\operatorname{dim} S_{\varepsilon}\right)-3=2 n-2$. Portanto, desde que $\lambda \leq n \leq 2 n-2$ (ou seja, $n \geq 2$ ), temos

$$
\pi_{1}\left(S_{\varepsilon} \backslash\left(D_{0}^{2 n} \cup e_{\lambda}\right)\right) \cong \pi_{1}\left(S_{\varepsilon} \backslash\left(D_{0}^{2 n}\right)=0 .\right.
$$

Mas, $\overline{F_{\theta}}$ é obtido de $D_{0}^{2 n}$ após a adjunção de um número finito de células. Portanto,

$$
0=\pi_{1}\left(S_{\varepsilon} \backslash D_{0}^{2 n}\right) \cong \pi_{1}\left(\overline{F_{\theta}}\right) .
$$

Usando o Corolário 4.0.27 obtemos

$$
0=\pi_{1}\left(\overline{F_{\theta}}\right) \cong \pi_{1}\left(S_{\varepsilon} \backslash \overline{F_{\theta}}\right) \cong \pi_{1}\left(F_{\theta}\right)
$$


Isto completa a demonstração.

Teorema 4.0.30. Cada fibra $F_{\theta}$ tem o tipo de homotopia de um buquê de esferas:

$$
S^{n} \vee S^{n} \vee \ldots \vee S^{n}
$$

Demonstração. Como consequência do Teorema 3.0.16. $H_{n}\left(F_{\theta}\right)$ é um grupo abeliano livre. Portanto, se $n \geq 2$, usando o Teorema de Hurewicz [26], temos que

$$
\pi_{n}\left(F_{\theta}\right) \cong H_{n}\left(F_{\theta}\right)
$$

Assim, cada gerador do grupo livre $\pi_{n}\left(F_{\theta}\right)$ é representado por uma aplicação contínua $\left(S^{n}, p_{0}\right) \longrightarrow\left(F_{\theta}, p_{1}\right)$ (representante da classe de homotopia) de maneira que estas aplicações determinam $\left(S^{n} \vee \ldots \vee S^{n}, p_{0}\right) \longrightarrow\left(F_{\theta}, p_{1}\right)$, uma aplicação contínua que leva o ponto base $p_{0}$ no ponto base $p_{1}$ e que leva as esferas $\left(S^{n}, p_{0}\right)$ do buquê nos geradores de $\pi_{n}\left(F_{\theta}, p_{1}\right)$. Uma tal aplicação induz um isomorfismo entre as homologias de tais espaços:

$$
H_{n}\left(S^{n} \vee \ldots \vee S^{n}\right) \cong H_{n}\left(F_{\theta}\right)
$$

O Teorema de Whitehead ([25 7.5.9) garante que a aplicação acima é uma equivalência de homotopia, já que $F_{\theta}$ e $S^{n} \vee \ldots \vee S^{n}$ são simplesmente conexos.

Se $\mathrm{n}=1$, temos o caso clássico. Neste caso, $\overline{F_{\theta}}$ é uma superfície conexa compacta orientável e com bordo. Pela classificação dessas variedades de dimensão 2 [11], segue que $\overline{F_{\theta}}$ tem o tipo de homotopia de um buquê de esferas $S^{1}$.

Definição 4.0.6. O número de esferas do buquê mencionado no Teorema 4.0.30 é denominado número de Milnor da aplicação $f$.

Observação 4.0.31. Os Teoremas 2.0.15 e 4.0.30 compõe o resultado que é popularmente conhecido como o Teorema da Fibração de Milnor. 



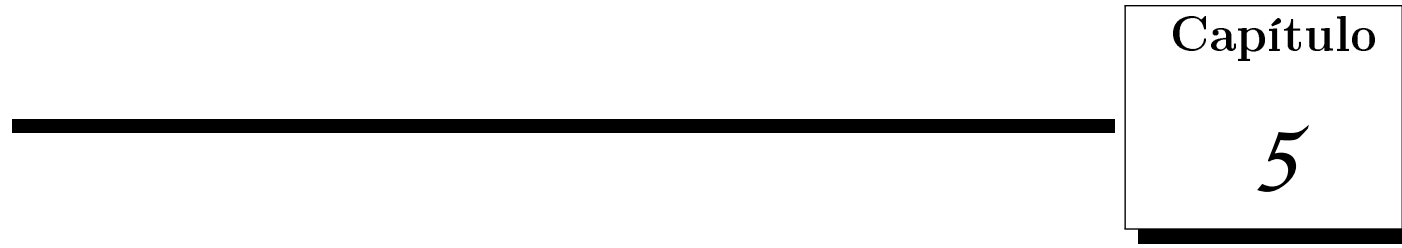

\section{Funções Analíticas Complexas com}

\section{Singularidades Isoladas}

Em [13], Lê D. T. investigou os espaços nos quais é válido um resultado similar ao resultado de Milnor que apresentamos nos capítulos anteriores. Ele estudou alguns espaços nos quais é possível obter uma generalização do Teorema da Fibração de Milnor para o caso de funções definidas em variedades singulares, de modo a caracterizar a topologia de uma fibra genérica na vizinhança do ponto singular.

Neste capítulo, nos propormos a apresentar os principais resultados de [13]. Por se tratar de um artigo denso demostraremos o teorema 5.1.3 e apenas mencionaremos as principais ferramentas utilizadas nas demonstrações dos resultados 5.2.1 e 5.4.1.

As principais referências para este Capítulo são [13, 14, 2].

\subsection{Funções com singularidades isoladas}

Começaremos a seção com as definições de fibrado cotangente e espaço conormal, ferramentas essenciais para nossos estudos ao longo deste capítulo. 
Definição 5.1.1. Seja $M$ uma variedade diferenciável de dimensão $m$. O conjunto

$$
T^{*}(M)=\bigcup_{p \in M}\{p\} \times\left(T_{p} M\right)^{*}
$$

de todos as formas lineares $\left(T_{p} M\right)^{*}$ definidas nos espaços tangente $T_{p} M$, com $p \in M$, "colados" de uma maneira natural é chamado de fibrado cotangente de $M$.

Definição 5.1.2. Seja $U \subset M$. O espaço conormal de $U$ em $M$ é o subespaço

$$
T_{U}^{*}(M)
$$

do espaço cotangente $T^{*}(M)$ das formas lineares que se anulam em $T_{p} U, \forall p \in U$.

Introduziremos a seguir o conceito de germe de funções analíticas complexas com singularidade isolada definido sobre um germe de espaço analítico complexo na origem, $(X, 0)$, com uma estratificação de Whitney $\left\{X_{i}\right\}_{i}$. Trabalharemos sempre com representantes $X$ equidimensionais e mergulhados em algum espaço $\mathbb{C}^{n}$.

Definição 5.1.3. Uma função analítica complexa $f: X \rightarrow \mathbb{C}$ definida sobre um espaço analítico complexo $X$, munido de uma estratificação de Whitney $\left\{X_{i}\right\}_{i}$, terá uma singularidade isolada em $0 \in X$ relativa a esta estratificação de Whitney, se existir uma vizinhança $U$ de 0 em $X$ tal que, para todo estrato $X_{i}$ para o qual $X_{i} \cap U \neq \varnothing$, a restrição de $f$ a $X_{i} \cap U \backslash\{0\}$ tem posto 1 .

Esta definição é equivalente à seguinte proposição:

Proposição 5.1.1. Uma função analítica complexa $f: X \rightarrow \mathbb{C}$ terá uma singularidade isolada na origem, $0 \in X$, relativa à estratificação de Whitney $\left\{X_{i}\right\}_{i}$, se existir uma vizinhança $U$ de 0 em $X$ e uma extensão $F$ a uma vizinhança aberta $\mathcal{U}$ de 0 em $\mathbb{C}^{n}$ da restrição $f \mid U$, tal que a interseção da imagem Im $d F$ no fibrado cotangente $T^{*}(\mathcal{U})$ da diferencial $d F: \mathcal{U} \rightarrow T^{*}(\mathcal{U})$ de $F$ e a união dos espaços conormais $\cup_{i} T_{X_{i} \cap \mathcal{U}}^{*}(\mathcal{U})$ for igual ao ponto $\left(0, d F_{0}\right)$ :

$$
\left\{\left(0, d F_{0}\right)\right\}=\boldsymbol{I} \boldsymbol{m} d F \cap\left(\bigcup_{i}\left(T_{X_{i} \cap \mathcal{U}}^{*}(\mathcal{U})\right) .\right.
$$


Teorema 5.1.2 (Teorema de Bertini-Sard, [27]). Se $\left\{X_{i}\right\}_{i}$ for uma estratificação de Whitney de $X$, para $\varepsilon>0$ suficientemente pequeno, a esfera $\partial B_{\varepsilon}(0)$ será transversal aos estratos $X_{i}$.

Uma consequência do Primeiro Teorema de Isotopia de Thom-Mather (ver [1]) juntamente com o Teorema de Bertini-Sard nos fornece o seguinte resultado:

Teorema 5.1.3. Seja $f: X \rightarrow \mathbb{C}$ uma função analítica complexa com singularidade isolada na origem de $X$, relativa à estratificação de Whitney $\left\{X_{i}\right\}_{i}$. Existe uma vizinhança $U$ de 0 em $X$ tal que existe $\varepsilon_{0}>0$ tal que, para todo $\varepsilon, \varepsilon_{0} \geq \varepsilon>0$, existe $\eta_{\varepsilon}>0$ tal que, para todo $\eta, \eta_{\varepsilon} \geq \eta>0$, a função $f$ induz uma aplicação $\varphi_{\varepsilon, \eta}$ de $B_{\varepsilon}(0) \cap X \cap f^{-1}\left(D_{\eta}(f(0))\right)$ sobre $D_{\eta}(f(0))$,

$$
\varphi_{\varepsilon, \eta}: B_{\varepsilon}(0) \cap X \cap f^{-1}\left(D_{\eta}(f(0))\right) \rightarrow D_{\eta}(f(0))
$$

que é uma fibração localmente trivial sobre o disco menos um ponto

$$
D_{\eta}^{*}(f(0)):=D_{\eta}(f(0)) \backslash\{f(0)\}
$$

Demonstração. As interseções não vazias dos estratos de $\left\{X_{i}\right\}_{i}$ com a vizinhança $U$ fornecem uma estratificação de Whitney de $U \cap X$.

Seja $U$ suficientemente pequeno, de maneira que as restrições de $f$ aos estratos de $U \cap X$ tenham posto 1, exceto possivelmente em 0 . Então, a fibra $U \cap f^{-1}(f(0))$ é estratificada por $\{0\}$ e pelas interseções não vazias dos estratos de $\left\{X_{i}\right\}_{i} \operatorname{com} U \cap f^{-1}(f(0))$ menos $\{0\}$. Vamos nos referir a estas estratificações de $U \cap X$ e $U \cap f^{-1}(f(0))$ como as estratificações induzidas por $\left\{X_{i}\right\}_{i}$.

Seja $\bar{B}_{\varepsilon}(0)$ a bola fechada em $\mathbb{C}^{n}$ centrada em 0 com raio $\varepsilon$. Se $\varepsilon>0$ for suficientemente pequeno, digamos $\varepsilon_{0} \geq \varepsilon>0$, para algum $\varepsilon_{0}$, então o Teorema de Bertini-Sard mostra que a esfera $S_{\varepsilon}(0)$ que limita $\bar{B}_{\varepsilon}(0)$ intersepta transversalmente os estratos de $\left\{X_{i}\right\}_{i}$ e os estratos de $U \cap f^{-1}(f(0))$ induzidos por $\left\{X_{i}\right\}_{i}$.

A estratificação de Whitney $\left\{X_{i}\right\}_{i}$ de $X$ induz uma estratificação de Whitney sobre $\bar{B}_{\varepsilon}(0) \cap X$ cujos estratos são as interseções não vazias dos estratos de $\left\{X_{i}\right\}_{i}$ com a bola aberta $B_{\varepsilon}(0)$ e com a esfera $S_{\varepsilon}(0)$, respectivamente. 
Novamente pelo Teorema de Bertini-Sard citado acima, para cada ponto de $S_{\varepsilon}(0) \cap$ $f^{-1}(f(0))$, existe uma vizinhança na qual as restrições de $f$ aos estratos de $S_{\varepsilon}(0) \cap X$ tem posto real 2. Como a interseção $S_{\varepsilon}(0) \cap X$ é compacta, existe $\eta_{\varepsilon}$ tal que, para todo $\eta$ satisfazendo $\eta_{\varepsilon} \geq \eta>0$, as restrições de $f$ aos estratos de $S_{\varepsilon}(0) \cap X$ tem posto real 2 nos pontos de $f^{-1}\left(D_{\eta}(f(0))\right)$. A aplicação $\bar{\varphi}_{\varepsilon, \eta}$, de $\bar{B}_{\varepsilon}(0) \cap X \cap f^{-1}\left(D_{\eta}(f(0))\right)$ sobre $D_{\eta}(f(0))$,

$$
\bar{\varphi}_{\varepsilon, \eta}: \bar{B}_{\varepsilon}(0) \cap X \cap f^{-1}\left(D_{\eta}(f(0))\right) \longrightarrow D_{\eta}(f(0))
$$

induzida por $f$ é portanto estratificada.

Como a aplicação $\bar{\varphi}_{\varepsilon, \eta}$ é própria, pelo Primeiro Teorema de Isotopia de Thom-Mather, ela é uma fibração contínua localmente trivial sobre o disco menos um ponto $D_{\eta}^{*}(f(0)):=$ $D_{\eta}(f(0)) \backslash\{f(0)\}$.

Por outro lado, as restrições de $f$ aos estratos de $S_{\varepsilon}(0) \cap X \cap f^{-1}\left(D_{\eta}(f(0))\right)$ tem posto máximo, e o Primeiro Teorema de Isotopia de Thom-Mather novamente nos diz que $f$ induz uma fibração de $S_{\varepsilon}(0) \cap X \cap f^{-1}\left(D_{\eta}(f(0))\right)$ sobre os disco $D_{\eta}(f(0))$. Sobre o disco furado, $D_{\eta}^{*}(f(0))$, ela é uma subfibração de $\bar{\varphi}_{\varepsilon, \eta}$. Portanto, a restrição $\varphi_{\varepsilon, \eta}$ de $\bar{\varphi}_{\varepsilon, \eta}$ a $B_{\varepsilon}(0) \cap X \cap f^{-1}\left(D_{\eta}(f(0))\right)$ é também uma fibração contínua localmente trivial sobre o disco furado $D_{\eta}^{*}(f(x))$.

\subsection{Morsificação}

Nesta seção vamos considerar uma função analítica complexa $F: \mathcal{U} \rightarrow \mathbb{C}$ definida sobre uma vizinhança aberta $\mathcal{U}$ de $0 \mathrm{em} \mathbb{C}^{n}$, e um germe de espaço analítico complexo fechado $X$ em $\mathcal{U}$. Denotaremos por $f$ a restrição de $F$ a $X$.

Assumiremos que $\left\{X_{i}\right\}_{i}$ é uma estratificação de Whitney analítica complexa de $X$ e que $f$ tem uma singularidade isolada em 0 relativa à esta estratificação. Definimos:

Definição 5.2.1. Diremos que 0 é um ponto de Morse complexo de $f$ relativo à estratificação de Whitney $\left\{X_{i}\right\}_{i}$, se a imagem, Im $d F$, da diferencial dF intersepta a união dos espaços conormais $\cup_{i} T_{X_{i}}^{*} \cap \mathcal{U}(\mathcal{U})$ transversalmente em $T^{*}(\mathcal{U})$, no ponto $\left(0, d F_{0}\right)$. 
Em particular, se o ponto 0 for um ponto de Morse complexo de $f$ relativo à estratificação $\left\{X_{i}\right\}_{i},\left(0, d F_{0}\right)$ não está contido no espaço conormal de $\mathcal{U}$ do fecho de qualquer estrato $\bar{X}_{i}$ de $\left\{X_{i}\right\}_{i}$, para $i \neq i_{0}$. Isto é,

$$
\left(0, d F_{0}\right) \notin \bigcup_{i \neq i_{0}} T_{\overline{X_{i}} \cap \mathcal{U}}^{*}(\mathcal{U}) .
$$

Portanto, ou $d F_{0}$ tem posto 1 e o núcleo, ker $d F_{0}$, é tangente ao estrato $X_{i_{0}}$ no ponto isolado 0 e não é um limite de hiperplanos tangentes aos fechos $\bar{X}_{i}$ dos estratos $X_{i}$ de $\left\{X_{i}\right\}_{i}$, para $i \neq i_{0}$, ou $d F_{0}$ tem posto 0 , isto é, $d F_{0}=0$, e necessariamente, em $\left(0, d F_{0}\right)=(0,0)$, o espaço conormal $T_{X_{i_{0}} \cap \mathcal{U}}^{*}(\mathcal{U})$ é a seção zero do fibrado cotangente e $X_{i_{0}}$ é uma vizinhança de 0 em $\mathbb{C}^{n}$. Neste caso, $F$ tem um ponto quadrático ordinário em 0.

Usando a notação acima temos o seguinte teorema:

Teorema 5.2.1. Existe um subconjunto aberto e denso $\Omega$ do espaço das formas lineares complexas de $\mathbb{C}^{n}$ tais que, para cada forma linear $l \in \Omega$, existe $\varepsilon_{0}>0$ tal que, para todo $\varepsilon$ satisfazendo $\varepsilon_{0} \geq \varepsilon>0$, existe $\tau_{\varepsilon}>0$ tal que, para todo $t$ com $\tau_{\varepsilon} \geq|t|>0$, a restrição a $X$ da função $F+t l$ tem apenas pontos de Morse complexos em $B_{\varepsilon}(0) \cap X$.

Na prova deste teorema, em [13] o autor define uma aplicação basicamente definida entre espaços cotangentes, usa fortemente o Teorema de Preparação de Weirstrass, resultados sobre recobrimento ramificado e que a imagem da interseção de $\mathbf{I m} d F$ com a união dos conormais aos estratos de $X$ é um ponto isolado.

Este teorema representa um resultado muito importante para a teoria de singularidades. Ele diz que, dada uma função analítica complexa com singularidade isolada como acima, é possível aproximá-la por funções analíticas complexas que admitem apenas singularidades do tipo Morse. Intuitivamente falando, uma singularidade do tipo Morse é uma singularidade "estável"no sentido de que é impossível "dividí-la"em mais singularidades. 


\subsection{Link Complexo e Profundidade}

\subsubsection{O Link Complexo}

No estudo de estratificações de Whitney analíticas complexas, M. Goresky e R. MacPherson introduziram o conceito de link complexo de um estrato de Whitney e mostraram que o link complexo é o elemento chave para uma teoria de Morse em espaços analíticos complexos [2]. Este espaço contém várias informações sobre o comportamento de $X$ em torno de um ponto $x$ escolhido, que em nosso caso será sempre a origem.

Seja $0 \in X \subset \mathbb{C}^{n}$, representante de um germe de espaço analítico complexo $(X, 0)$.

Definição 5.3.1. Para $l: \mathbb{C}^{n} \longrightarrow \mathbb{C}$ função linear genérica e $\varepsilon>0$ suficientemente pequeno, definimos o link complexo de $X$ em 0 como o espaço

$$
\mathcal{L}_{0}:=B_{\epsilon}(0) \cap X \cap l^{-1}(t)
$$

em que $t \neq l(0)$ está suficientemente próximo de $l(0)$.

Teorema 5.3.1 ([2, 16]). Existe um aberto de Zariski $\Omega$ no espaço das formas lineares complexas de $\mathbb{C}^{n}$ tal que, para todo $l \in \Omega$, existe $\varepsilon_{l}>0$ de modo que, para qualquer $\varepsilon$, $\varepsilon_{l}>\varepsilon>0$, existe um $\tau_{\varepsilon, l}$ tal que, para qualquer $t$ com $\tau_{\varepsilon, l}>|t|>0$, o tipo de homotopia do link complexo

$$
\mathcal{L}_{0}:=B_{\varepsilon}(0) \cap X \cap l^{-1}(l(0)+t)
$$

é um invariante analitico do germe $(X, 0)$.

Considere $(X, 0)$ um germe de espaço analítico complexo e $X$ um representante suficientemente pequeno, Whitney estratificado como anteriormente, e 0 um ponto de $X$. Seja $X_{0}$ o estrato que contém 0 e $N$ um subespaço afim de $\mathbb{C}^{n}$ passando por 0 , de codimensão igual à dimensão do estrato $X_{0}$ e transversal ao estrato em 0 . Ou seja, $N$ é tal que $N \cap X_{0}=\{0\}$. 
Definição 5.3.2. O link complexo do conjunto $X \cap N$ em 0 é chamado de link complexo do estrato $X_{0}$ em $X$ e é denotado por $\mathcal{L}_{X_{0}}$.

Teorema 5.3.2 ([2, 16]). O tipo de homotopia do link complexo do estrato é um invariante analitico do germe $(X, 0)$ e não depende da escolha do ponto $x$ no estrato.

Definição 5.3.3. Nas condições anteriores, para $\varepsilon$ suficientemente pequeno, o espaço $X \cap N \cap B_{\varepsilon}(0)$ é chamado de uma fatia normal $\mathcal{N}_{0}$ em $X$ de $X_{0}$ em 0 , e o par $(\mathcal{N}, \mathcal{L})$ é chamado de dados normais de Morse do estrato $X_{0}$ em $X$.

\subsubsection{Profundidade homotópica retificada}

Nosso interesse na profundidade homotópica retificada (rhd) vem do fato que medindo a rhd para espaços analíticos complexos obtemos informações sobre seus dados normais de Morse.

A seguinte definição é atribuída à Grothendieck [5].

Definição 5.3.4. Sejam $(X, 0)$ germe de um espaço analítico complexo, $X$ um representante suficientemente pequeno do germe e $Y$ um subconjunto analitico fechado de $X$. Diremos que $X$ tem profundidade homotópica $h d_{Y}(X) \geq n$ ao longo de $Y$ se, para qualquer $y \in Y$, existir um sistema fundamental de vizinhanças $\left\{U_{\alpha}\right\}_{\alpha}$ de y em $X$ tal que os pares $\left(U_{\alpha}, U_{\alpha}-Y\right)$ sejam $(n-1)-$ conexos.

$O$ número inteiro $h d_{Y}(X)$ é definido como sendo o supremo do conjunto dos inteiros n como na definição acima.

Definição 5.3.5. Sejam $(X, 0)$ e $X$ como anteriormente, $x$ um ponto de $X$ e $Y \subset X$ um subespaço analítico complexo de $X$. Assuma que $x \in Y$. Dizemos que o espaço $X$ (ou o germe $(X, 0)$ ) tem profundidade homotópica $h d_{Y}(X, 0) \geq n$ ao longo de $Y$ no ponto $x$ se existe uma vizinhança aberta $U$ de $x$ em $X$ tal que a profundidade homotópica $h d_{Y \cap U}(X \cap U)$ de $(X \cap U)$ ao longo de $Y \cap U$ seja maior ou igual a $n$.

Lema 5.3.3. Seja $\left\{X_{i}\right\}$ uma estratificação de Whitney de $X$. A função $h d_{X_{i}}(X, 0)$ é constante ao longo dos estratos $X_{i}$ de $X$.

Podemos agora, definir a noção de profundidade homotópica retificada. 
Definição 5.3.6. Dizemos que a profundidade homotópica retificada $\operatorname{rhd}(X, x)$ de $X$ em um ponto $x$ é maior ou igual a $n$ se, para qualquer subespaço analítico fechado $Y$ de $X$, existe uma vizinhança $U$ de $x$ em $X$ tal que a profundidade homotópica de $X \cap U$ ao longo de $Y \cap U$ seja maior ou igual a $n-\operatorname{dim} Y$.

O inteiro $\operatorname{rhd}(X, x)$ é o supremo do conjunto de inteiros $n$ como na definição anterior.

Observação 5.3.4. Note que $\operatorname{rhd}(X, x) \leq \operatorname{dim}(X)$. De fato, se $Y=X$ e U é uma boa vizinhança (ver [8]) de $x$ em $X$, o par $(U, U-X)$ não é 0 -conexo, pois $U-X=\varnothing$.

Os links complexos de uma estratificação de Whitney complexa fornecem uma boa caracterização para profundidade homotópica retificada:

Teorema 5.3.5. Seja $(X, 0)$ germe de um espaço analítico complexo, $X$ um representante suficientemente pequeno do germe e $x \in X$. Seja $\left\{X_{i}\right\}$ uma estratificação de Whitney de $X$. As seguintes condições são equivalentes:

1. $\operatorname{rhd}(X, x) \geq n$;

2. Para qualquer estrato $X_{i}$ de $\left\{X_{i}\right\}$ que contenha o ponto $x$ em seu fecho, os dados normais de Morse $(\mathcal{N}, \mathcal{L})$ de $X_{i}$ em $X$ é $\left(n-\operatorname{dim} X_{i}-1\right)$-conexo.

Observação 5.3.6. Este teorema é muito útil quando tomamos uma estratificação de Whitney simples do espaço $X$. Por exemplo, suponha que $X$ tenha uma singularidade isolada em 0 . Então, $\operatorname{rhd}(X, 0) \geq n$ se, e somente se, o link complexo de $\{0\}$ em $X$ for $(n-2)$-conexo. De fato, a condição $(c)$ do Teorema 1.4 de [8] mostra que $\operatorname{rhd}(X, 0) \geq n$ se, e somente se, existe um sistema fundamental de vizinhanças $\left\{U_{\alpha}\right\}_{\alpha}$ de 0 em $X$ tal que $\left(U_{\alpha}, U_{\alpha}-\{0\}\right)$ é $(n-1)$-conexo. Como estas vizinhanças podem ser substituídas por boas vizinhanças (veja [8]), pode-se considerar o sistemas fundamental de boas vizinhanças de $x$ em $X$ dado por $B_{\varepsilon}(0) \cap X$, com $\varepsilon>0$ suficientemente pequeno. Nesta situação, pela estrutura cônica local da singularidade, o par $\left(B_{\varepsilon}(0) \cap X, B_{\varepsilon}(0) \cap X-\{0\}\right)$ é $(n-1)$-conexo se, e somente se, o link algébrico $S_{\varepsilon}(x) \cap X$ da singularidade $x$ em $X$ é $(n-2)$-conexo.

Imediatamente, obtemos o corolário: 
Corolário 5.3.7. Seja $(X, 0)$ germe de um espaço analítico complexo, $X$ um representante suficientemente pequeno do germe e $x \in X$. Seja $\left\{X_{i}\right\}$ uma estratificação de Whitney de $X$. As seguintes condições são equivalentes:

1. $\operatorname{rhd}(X, x)=\operatorname{dim} X$;

2. Para qualquer estrato $X_{i}$ de $\left\{X_{i}\right\}$ que contenha o ponto $x$ em seu fecho, o link complexo $\mathcal{L}$ de $X_{i}$ em $X$ tem o tipo de homotopia de um buquê de esferas de dimensão real igual à dimensão complexa de $\mathcal{L}$.

Observação 5.3.8. Para simplificar, quando um link complexo $\mathcal{L}$ de $X_{i}$ em $X$ tiver o tipo de homotopia de um buquê de esferas de dimensão real igual à dimensão complexa de $\mathcal{L}$, diremos que $\mathcal{L}$ tem o tipo de homotopia de um buquê de esferas de dimensão média.

\subsection{O número de Milnor de Lê}

Concluímos este trabalho com a generalização do resultado de Milnor, apresentada por Lê D. T. em [13], sobre as fibras de funções analíticas complexas com singularidades isoladas.

Teorema 5.4.1. [13, 14] Sejam $(X, 0)$ um germe de espaço analitico complexo e $X$ um representante do germe suficientemente pequeno. Considere uma função analítica complexa $f$ definida em $X$ e suponha que ela tenha uma singularidade isolada em 0. Então, se $\operatorname{rhd}(X, 0)=\operatorname{dim} X$, uma fibra genérica de $f$ em 0 tem o tipo de homotopia de um buquê de esferas de dimensão real $\operatorname{dim} X-1$.

A principal ferramenta na demostração do teorema anterior é a teoria de Morse estratificada de M. Goresky e R. MacPherson [2]. Além disso, os conceitos de transversalidade e $q$-conexidade são fortemente utilizados.

Definição 5.4.1. Sejam $(X, 0)$ um germe de espaço analítico complexo, $X$ um representante do germe suficientemente pequeno e $f$ uma função analítica complexa definida em X, com singularidade isolada em 0 . O número de esferas do buquê mencionado no Teorema 5.4.1 é denominado número de Milnor de Lê de $f$. 
Definição 5.4.2. Estes espaços sobre os quais Lê D. T. pôde construir tal resultado, isto é, os espaços nos quais rhd $(X, 0)=\operatorname{dim} X$ foram denominados Espaços de Milnor. 


\section{Referências Bibliográficas}

[1] C. G. Gibson, K. Wirthmüller, A. A. du Plessis, and E. J. N. Looijenga. Topological stability of smooth mappings. Lecture Notes in Mathematics, Vol. 552. Springer-Verlag, Berlin, 1976.

[2] M. Goresky and R. MacPherson. Stratified Morse theory, volume 14 of Ergebnisse der Mathematik und ihrer Grenzgebiete (3) [Results in Mathematics and Related Areas (3)]. Springer-Verlag, Berlin, 1988.

[3] M. J. Greenberg. Lectures on algebraic topology. W. A. Benjamin, Inc., New York-Amsterdam, 1967.

[4] P. Griffiths and J. Harris. Principles of algebraic geometry. Wiley Classics Library. John Wiley \& Sons Inc., New York, 1994. Reprint of the 1978 original.

[5] A. Grothendieck. Cohomologie locale des faisceaux cohérents et théorèmes de Lefschetz locaux et globaux (SGA 2). North-Holland Publishing Co., Amsterdam, 1968. Augmenté d'un exposé par Michèle Raynaud, Séminaire de Géométrie Algébrique du Bois-Marie, 1962, Advanced Studies in Pure Mathematics, Vol. 2.

[6] R. C. Gunning and H. Rossi. Analytic functions of several complex variables. AMS Chelsea Publishing, Providence, RI, 2009. Reprint of the 1965 original.

[7] H. Hamm. Lokale topologische Eigenschaften komplexer Räume. Math. Ann., 191:235-252, 1971.

[8] H. A. Hamm and D. T. Lê. Rectified homotopical depth and Grothendieck conjectures. In The Grothendieck Festschrift, Vol. II, volume 87 of Progr. Math., pages 311-351. Birkhäuser Boston, Boston, MA, 1990.

[9] R. Hartshorne. Algebraic geometry. Springer-Verlag, New York, 1977. Graduate Texts in Mathematics, No. 52 .

[10] A. Hatcher. Algebraic topology. Cambridge University Press, 2002.

[11] M. W. Hirsch. Differential topology. Springer-Verlag, New York, 1976. Graduate Texts in Mathematics, No. 33 . 
[12] D. T. Lê. Le concept de singularité isolée de fonction analytique. In Complex analytic singularities, volume 8 of Adv. Stud. Pure Math., pages 215-227. North-Holland, Amsterdam, 1987.

[13] D. T. Lê. Complex analytic functions with isolated singularities. J. Algebraic Geom., 1(1):83-99, 1992.

[14] D. T. Lê. Hypersurfaces and polar curves. Preprint 2010.

[15] D. T. Lê. Sur les cycles évanouissants des espaces analytiques. C. R. Acad. Sci. Paris Sér. A-B, 288(4):A283-A285, 1979.

[16] D. T. Lê and B. Teisser. Cycles évanouissants, sections planes et conditions de whitney. 40:65-103, 1983.

[17] E.L. Lima. Grupo fundamental e espaços de recobrimento. Instituto de Matemática Pura e Aplicada do C.N.Pq., 1977.

[18] J. Milnor. Morse theory. Based on lecture notes by M. Spivak and R. Wells. Annals of Mathematics Studies, No. 51. Princeton University Press, Princeton, N.J., 1963.

[19] J. Milnor. Singular points of complex hypersurfaces. Annals of mathematics studies. Princeton University Press, 1968.

[20] M. Morse. The calculus of variations in the large. Colloquium Publications - American Mathematical Society. American Mathematical Society, 1978.

[21] J. R. Munkres. Elements of algebraic topology. Addison-Wesley Publishing Company, Menlo Park, CA, 1984 .

[22] V. M. Nascimento. A Fibração de Milnor. Master's thesis, Universidade de São Paulo - Instituto de Ciências Matemáticas e de Computação, 1990.

[23] B. Nils. The Gramian and K-Volume in N-Space: Some Classical Results in Linear Algebra. Journal of Young Investigators, 2, 1999.

[24] G. Prando. O Teorema da Fibração de Milnor e a Topologia das Fibras. Master's thesis, Universidade Federal do Espírito Santo - Centro de Ciências e Exatas, 2011.

[25] Edwin H. Spanier. Algebraic topology. McGraw-Hill Book Co., New York, 1966.

[26] N. Steenrod. The Topology of Fibre Bundles. Princeton Mathematical Series, vol. 14. Princeton University Press, Princeton, N. J., 1951.

[27] J. L. Verdier. Stratifications de Whitney et théorème de Bertini-Sard. Invent. Math., 36:295-312, 1976.

[28] H. Whitney. Tangents to an analytic variety. Ann. of Math. (2), 81:496-549, 1965. 